\title{
Papers
}

\section{Collaborative meta-analysis of randomised trials of antiplatelet therapy for prevention of death, myocardial infarction, and stroke in high risk patients}

Antithrombotic Trialists' Collaboration

\begin{abstract}
Objective To determine the effects of antiplatelet therapy among patients at high risk of occlusive vascular events.

Design Collaborative meta-analyses (systematic overviews).

Inclusion criteria Randomised trials of an antiplatelet regimen versus control or of one antiplatelet regimen versus another in high risk patients (with acute or previous vascular disease or some other predisposing condition) from which results were available before September 1997. Trials had to use a method of randomisation that precluded prior knowledge of the next treatment to be allocated and comparisons had to be unconfounded-that is, have study groups that differed only in terms of antiplatelet regimen.

Studies reviewed 287 studies involving 135000 patients in comparisons of antiplatelet therapy versus control and 77000 in comparisons of different antiplatelet regimens.

Main outcome measure "Serious vascular event": non-fatal myocardial infarction, non-fatal stroke, or vascular death.

Results Overall, among these high risk patients, allocation to antiplatelet therapy reduced the combined outcome of any serious vascular event by about one quarter; non-fatal myocardial infarction was reduced by one third, non-fatal stroke by one quarter, and vascular mortality by one sixth (with no apparent adverse effect on other deaths). Absolute reductions in the risk of having a serious vascular event were 36 (SE 5) per 1000 treated for two years among patients with previous myocardial infarction; 38 (5) per 1000 patients treated for one month among patients with acute myocardial infarction; 36 (6) per 1000 treated for two years among those with previous stroke or transient ischaemic attack; 9 (3) per 1000 treated for three weeks among those with acute stroke; and 22 (3) per 1000 treated for two years among other high risk patients (with separately significant results for those with stable angina $(\mathrm{P}=0.0005)$, peripheral arterial disease $(\mathrm{P}=0.004)$, and atrial fibrillation $(\mathrm{P}=0.01))$. In each of these high risk categories, the absolute benefits substantially outweighed the absolute risks of major extracranial
\end{abstract}

bleeding. Aspirin was the most widely studied antiplatelet drug, with doses of 75-150 mg daily at least as effective as higher daily doses. The effects of doses lower than $75 \mathrm{mg}$ daily were less certain. Clopidogrel reduced serious vascular events by $10 \%$ $(4 \%)$ compared with aspirin, which was similar to the $12 \%(7 \%)$ reduction observed with its analogue ticlopidine. Addition of dipyridamole to aspirin produced no significant further reduction in vascular events compared with aspirin alone. Among patients at high risk of immediate coronary occlusion, short term addition of an intravenous glycoprotein IIb/IIIa antagonist to aspirin prevented a further 20 (4) vascular events per $1000(\mathrm{P}<0.0001)$ but caused 23 major (but rarely fatal) extracranial bleeds per 1000 . Conclusions Aspirin (or another oral antiplatelet drug) is protective in most types of patient at increased risk of occlusive vascular events, including those with an acute myocardial infarction or ischaemic stroke, unstable or stable angina, previous myocardial infarction, stroke or cerebral ischaemia, peripheral arterial disease, or atrial fibrillation. Low dose aspirin (75-150 $\mathrm{mg}$ daily) is an effective antiplatelet regimen for long term use, but in acute settings an initial loading dose of at least $150 \mathrm{mg}$ aspirin may be required. Adding a second antiplatelet drug to aspirin may produce additional benefits in some clinical circumstances, but more research into this strategy is needed.

\section{Introduction}

Previous meta-analyses of randomised trials have shown that antiplatelet therapy prevents serious vascular events, ${ }^{1}$ arterial occlusion, ${ }^{2}$ and venous thrombo$\mathrm{embolism}^{3}$ among a wide range of patients at high risk of occlusive vascular events. The proportional reduction in serious vascular events (non-fatal myocardial infarction, non-fatal stroke, or death from a vascular cause) was about one quarter in a wide range of high risk patients, irrespective of why the risk was high and irrespective of age, sex, blood pressure, or history of diabetes. $^{1}$

The previous meta-analyses, however, left some important clinical questions unanswered. For instance, although long term antiplatelet therapy was shown to be of substantial benefit after ischaemic stroke, it was

\section{Editorial by FitzGerald \\ Correspondence to: Antithrombotic Trialists' Secretariat, Clinical Trial Service Unit Radcliffe Infirmary, Oxford OX2 6HE www.ctsu.ox.ac.uk}

BMJ 2002;324:71-86

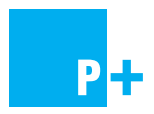

Details of the studies that were included, names of the collaborators, and a figure showing analyses of the proportional effects of treatment for different outcomes are available on bmj.com 
not known whether antiplatelet drugs were of net benefit as an immediate treatment in the acute phase of such strokes. ${ }^{4}$ There was also some uncertainty about whether antiplatelet therapy was of net benefit in patients with chronic conditions such as atrial fibrillation, stable angina, and atherosclerotic peripheral arterial disease that had been less extensively studied. Daily doses of at least $75 \mathrm{mg}$ of aspirin had been shown to be effective in long term use, but theoretical advantages had been proposed for lower doses. ${ }^{5}$

The previous meta-analyses included only those trials that were available in 1990, and since then there have been many additional trials of aspirin at various doses and of other antiplatelet drugs. ${ }^{6} 7$ There have also been trials of the effects of adding to aspirin another antiplatelet drug with a different mechanism of action. In addition, although certain anticoagulant regimens were known to be effective for particular high risk patients in the absence of antiplatelet therapy, it was not known whether the addition of anticoagulants to antiplatelets would provide additional protection. We have therefore updated previous meta-analyses to include studies available by September 1997. This paper summarises the updated results from the trials of antiplatelet drugs among high risk patients.

\section{Methods}

The methods and definitions used in the present metaanalysis were broadly similar to those used in the previous meta-analysis. ${ }^{1}$

\section{Identification of trials}

Details of each trial included in the analysis are available on bmj.com. The aim was to identify all trials, published or otherwise, that were available by September 1997 and that compared an antiplatelet regimen with a control or one antiplatelet regimen with another among patients considered to be at high annual risk (for example, over $3 \%$ a year) of vascular events because of evidence of pre-existing disease (previous occlusive event or predisposing condition). We included only those trials that were believed to have used a randomisation method that precluded prior knowledge of the next treatment to be allocated (thus, alternation or odd or even dates would not suffice) and were "unconfounded"-that is, contained two randomised groups that differed only with respect to the antiplatelet comparison of interest. Trials of oral antiplatelet regimens were eligible only if they had assessed more than one day of treatment, but we included trials of parenteral antiplatelet regimens of any duration. An antiplatelet drug was defined as one whose primary effect on the vascular system is to inhibit platelet adhesion, platelet aggregation, or both. ${ }^{1}$

We identified relevant trials by searching several electronic databases (Medline, Embase, Derwent, Scisearch, and Biosis; search strategy available on request); searching the trials registers of the Cochrane Stroke and Peripheral Vascular Disease Groups; manual searching of journals, abstracts, and proceedings of meetings; scrutinising the reference lists of trials and review articles; and inquiry among many colleagues, including representatives of pharmaceutical companies.

\section{Definition of outcomes}

The primary measure of outcome was a "serious vascular event" (that is, non-fatal myocardial infarction, non-fatal stroke, or death from a vascular cause and including any death from an unknown cause because most deaths in high risk patients are likely to be due to vascular causes). In order to allow the number of serious vascular events to be derived by adding the numbers of non-fatal myocardial infarctions, non-fatal strokes, and vascular deaths, we considered an event non-fatal only if the patient survived to the end of the scheduled follow up period (or died of a definitely non-vascular cause). Each contributing trialist's definition of a particular outcome (such as myocardial infarction) was used for counting vascular events, and we included all events classified by the trialist as probable or definite.

Deaths were divided into those with a vascular cause (defined as cardiac, cerebrovascular, venous thromboembolic, haemorrhagic, other vascular, or unknown cause) and those that were considered definitely non-vascular. Strokes were subdivided into intracranial haemorrhages (including intracerebral, subdural, subarachnoid, and extradural haemorrhages) and strokes of ischaemic or unknown aetiology; transient ischaemic attacks were not to be included. Major extracranial bleeds were those occurring outside the cranial cavity that were considered by the trialist to be serious (which, in general, meant that the patient required admission to hospital or blood transfusion). If during the trial a patient experienced more than one type of non-fatal outcome-for example, a myocardial infarction followed by a stroke-both events were recorded, but such patients contributed only once to the composite outcome of serious vascular event. If during the trial a patient experienced more than one non-fatal event of the same type (for example, two myocardial infarctions) or more than one pathological type of stroke (for example, a haemorrhagic stroke and an ischaemic stroke), only the first was to be recorded.

\section{Data requested}

We asked the coordinators of all potentially eligible trials for details about method of randomisation, blinding of treatment allocation, scheduled duration of treatment, and, if different, scheduled duration of follow up. Investigators for trials that had randomised at least 200 patients were asked to contribute, for each patient originally randomised, data on baseline characteristics (age, sex, blood pressure, and medical history) and dates of randomisation, follow up, and any vascular events that had occurred. In addition, we asked them for a tabular summary of the numbers of patients originally allocated to each treatment group (that is, without any post-randomisation exclusions) and the numbers of patients experiencing particular outcomes during the scheduled follow up period. These outcomes were non-fatal myocardial infarction, nonfatal stroke (haemorrhagic or other), non-fatal or fatal pulmonary embolism, death from a vascular or unknown cause, death from a definitely non-vascular cause, and major extracranial bleeding. Investigators responsible for trials that had randomised fewer than 200 patients were asked only for the tabular summary of the numbers of patients and outcomes (although a few such studies did contribute individual patient data). 
In trials assessing a month or more of treatment, we intended that analyses would be of events occurring during the scheduled treatment period, but in two trials follow up data were available only for a period in excess of the scheduled treatment period (see bmj.com for details). ${ }^{89}$ In trials with shorter courses of treatment, we analysed events during a period as close as possible to one month after randomisation. We checked data both for internal consistency and for consistency with relevant published reports and referred queries back to trial coordinators. Especially when data on individual patients were provided, the calculated numbers of vascular events may differ slightly from those reported in trial publications. Occasionally, when trial data had been discarded by investigators or were otherwise not available, the numbers of vascular events could be determined only from published reports.

\section{Statistical methods}

Proportional and absolute effects of treatment

We stratified analyses by trial to avoid direct comparisons between individuals in different studies. We calculated the observed minus the expected number of events, and its variance, from standard $2 \times 2$ tables of outcome by treatment. These were then summed over trials to give the grand total for observed minus expected events $(\mathrm{O}-\mathrm{E})$ and its variance $(\mathrm{V})$. We then based significance tests on comparison of $\mathrm{z}=(\mathrm{O}-\mathrm{E}) / \sqrt{ } \mathrm{V}$ with the standard normal distribution; $\mathrm{P}$ denotes the two sided significance level and $\mathrm{P}>0.05$ is non-significant by convention. The typical odds ratio for these trials was calculated by the one step method ${ }^{10}$ from $\mathrm{b}=(\mathrm{O}-\mathrm{E}) / \mathrm{V}$, either as $\exp (\mathrm{b})$ or, for rare events, as $(2+\mathrm{b}) /(2-\mathrm{b})$. For odds ratios between 0.5 and 2 these two methods give almost identical answers.

Some trials used a deliberately unequal randomisation ratio and so had a substantial imbalance in the numbers of patients in treatment and control groups. We multiplied the control group in such trials by an appropriate integer ${ }^{1}$ when calculating "adjusted" control totals (although not when making other calculations). When comparing the percentages affected in the treatment and in the adjusted control groups, we calculated the standard error (SE) of the difference (D) between these percentages as $\mathrm{D} / \mathrm{z}$.

\section{Effects in specific categories of trials}

We compared different trials or groups of trials using standard $\chi^{2}$ tests for heterogeneity or, where appropriate, tests for trend between the observed effects on vascular events (with appropriate allowance made for multiple comparisons). But, even where there is significant heterogeneity, groups of patients in whom treatment is particularly advantageous or relatively ineffective can be difficult to identify reliably. Especially when small numbers of patients in a particular category have been studied, it is important that "lack of evidence of benefit" when that category is considered on its own is not misinterpreted as "evidence of lack of benefit."11 As antiplatelet therapy reduces vascular events in a wide range of patients at high risk of occlusive vascular disease, the relevant question in any particular category is whether there is convincing evidence that there is no material benefit from treatment. ${ }^{12} 13$
Table 1 Major changes in availability of data between previous and current meta-analyses

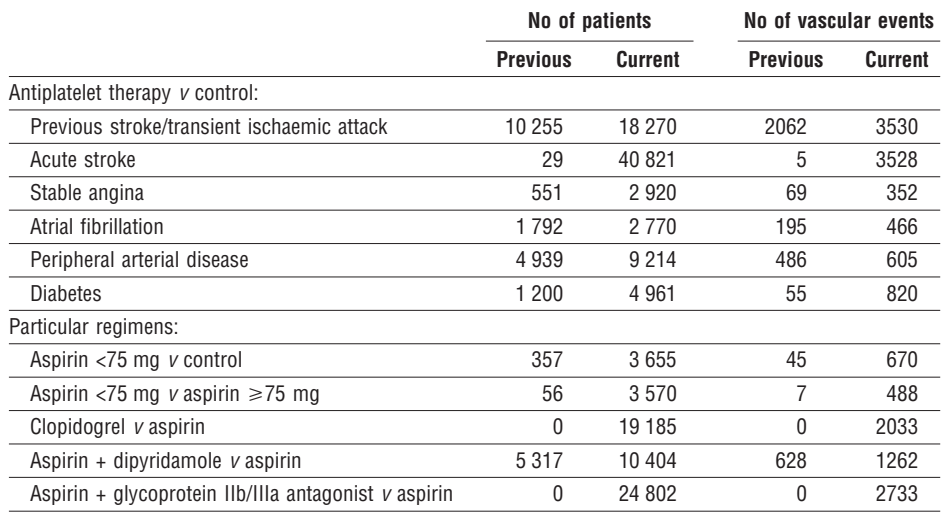

\section{Description of trials}

We identified 448 apparently randomised trials comparing an antiplatelet regimen with a control or one antiplatelet regimen with another among high risk patients. After review and, in cases of doubt, consultation with trial coordinators, 166 trials were excluded: 52 were not properly randomised, 24 were confounded, three had large numbers lost to follow up, 13 were abandoned before any outcome data were collected, 20 had a crossover design, and 54 had not systematically recorded any of the relevant outcome events. In addition, since the focus of the present analyses was on patients at high risk of occlusive arterial disease, we excluded trials among patients with dementia or occluded retinal veins (even if they had been included in the 1994 meta-analysis ${ }^{1}$ ). Insufficient information was available from 19 eligible trials among 3427 patients.

Details of the remaining 197 randomised trials that compared antiplatelet therapy versus control (195 with data on vascular events) and the 90 that compared different antiplatelet regimens (89 with data on vascular events) are available on bmj.com. Information on individual patients was available for trials that collectively included $59 \%$ of the vascular events, and in these trials fewer than $2 \%$ of patients were lost to follow up. (Further details of excluded trials and missing data are available on request.)

\section{Results}

Effects on serious vascular events among high risk patients

Information about serious vascular events (non-fatal myocardial infarction, non-fatal stroke, or vascular death) was available from 195 trials of antiplatelet treatment versus control among a total of 135640 patients at high risk of occlusive arterial disease (compared with 142 among 68814 previously ${ }^{1}$ ). There was substantial additional information about patients with a history of stroke or transient ischaemic attack, those treated early after an acute stroke, and those with stable angina, atrial fibrillation, peripheral arterial disease, or diabetes (table 1).

Overall, 7705 (10.7\%) serious vascular events were recorded among 71912 high risk patients allocated antiplatelet therapy versus an adjusted total of 9502 $(13.2 \%)$ among 72139 allocated control $(\mathrm{P}<0.0001$ : fig 1). When we subdivided the trials into five main 


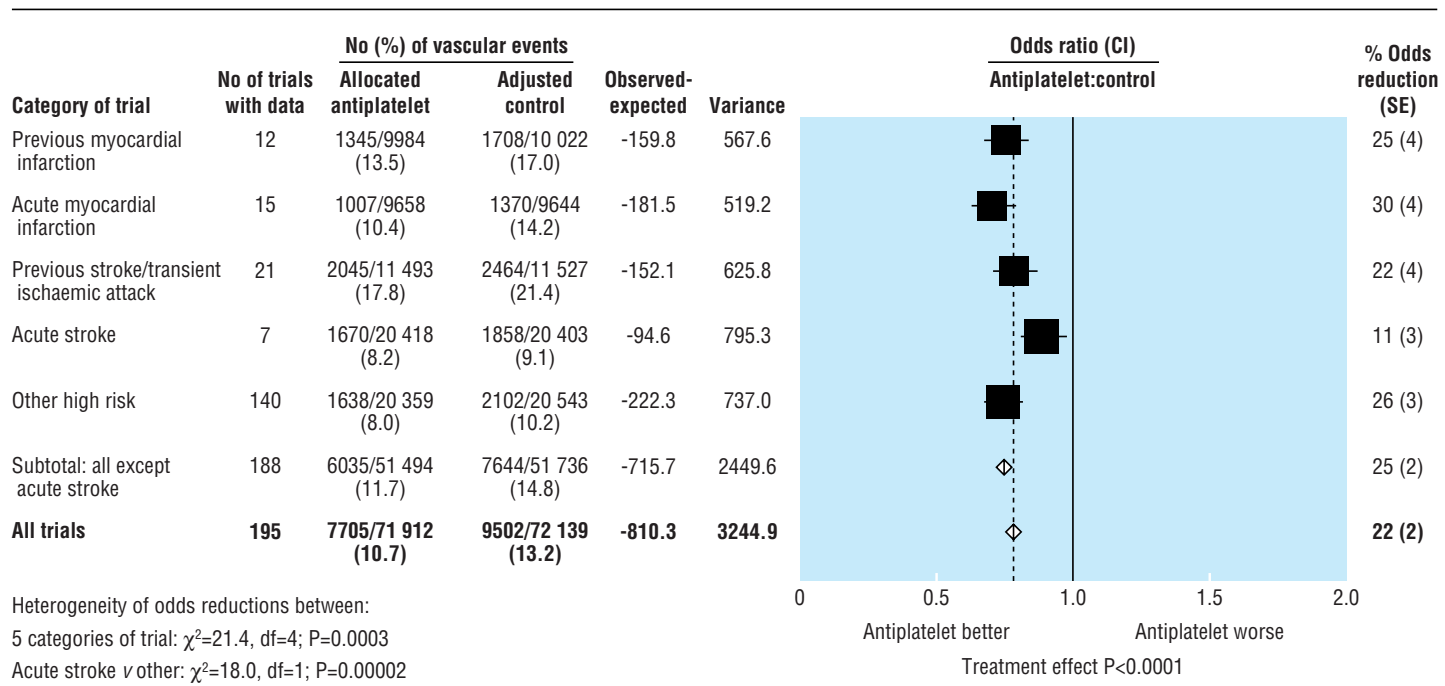

Fig 1 Proportional effects of antiplatelet therapy on vascular events (myocardial infarction, stroke, or vascular death) in five main high risk categories. Stratified ratio of odds of an event in treatment groups to that in control groups is plotted for each group of trials (black square) along with its $99 \%$ confidence interval (horizontal line). Meta-analysis of results for all trials (and 95\% confidence interval) is represented by an open diamond. Adjusted control totals have been calculated after converting any unevenly randomised trials to even ones by counting control groups more than once, but other statistical calculations are based on actual numbers from individual trials

categories of high risk patient, there was clear evidence that the proportional reductions in serious vascular events differed among them $\left(\chi^{2}\right.$ for heterogeneity between these categories $=21.4, \mathrm{df}=4 ; \mathrm{P}=0.0003)$, mainly because of the somewhat smaller effect observed in patients treated during acute stroke $\left(\chi^{2}\right.$ for heterogeneity between acute stroke and other categories $=18.0$, $\mathrm{df}=1 ; \mathrm{P}=0.00002)$. Even so, the net benefit was highly significant both among patients with acute stroke $(\mathrm{P}=0.0009)$ and, separately, among patients in each of the other high risk categories (each $\mathrm{P}<0.0001$ ).

Among patients with acute stroke, the absolute reduction in the risk of a serious vascular event was 9 (SE 3) per 1000 patients allocated antiplatelet therapy. This is smaller than the absolute benefit of 22 to 38 fewer vascular events per 1000 among the other four categories of high risk patients (fig 2). However, the net

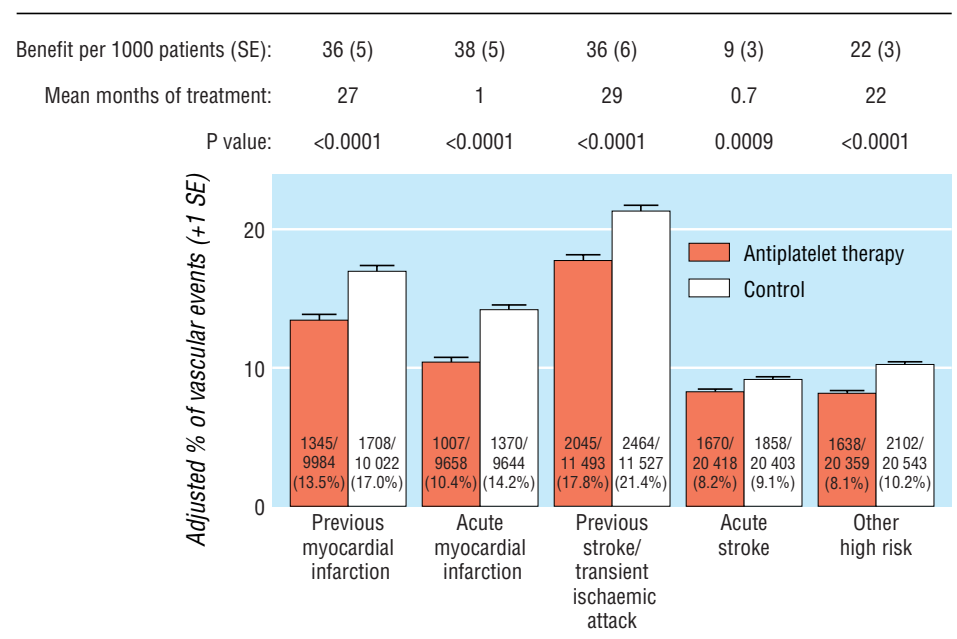

Fig 2 Absolute effects of antiplatelet therapy on vascular events (myocardial infarction, stroke, or vascular death) in five main high risk categories. Adjusted control totals have been calculated after converting any unevenly randomised trials to even ones by counting control groups more than once benefit in patients with acute stroke was achieved with less than one month of treatment, whereas the benefit among patients with a previous stroke or transient ischaemic attack (36 fewer events per 1000) resulted from an average of 29 months of treatment. Thus, the net benefit per month of antiplatelet treatment is substantially greater in the first month (starting at the time of the acute stroke) than it is during long term treatment for secondary prevention of stroke. Among patients with high risk conditions other than acute stroke, antiplatelet treatment produced a 25\% (SE 2\%) proportional reduction in serious vascular events that was similar in each of the four subcategories studied $\left(\chi^{2}=3.4, \mathrm{df}=3\right.$; NS, fig 1$)$.

\section{Effects on different measures of outcome among high risk patients}

Non-fatal myocardial infarction as outcome

Information was available on 2774 non-fatal myocardial infarctions after randomisation in 159 trials among high risk patients (compared with 2199 in 120 trials previously ${ }^{1}$ ) and on a further 4828 deaths attributed to coronary heart disease. Data for non-fatal myocardial infarctions are in figures 3a-3c (but had not been sought by the investigators in the main trials among acute stroke patients). Overall, antiplatelet treatment produced a 34\% (3\%) proportional reduction in non-fatal myocardial infarction $(\mathrm{P}<0.0001$; see figure on bmj.com) and a $26 \%(2 \%)$ reduction in nonfatal myocardial infarction or death from coronary heart disease $(\mathrm{P}<0.0001)$. In each of the four categories of trial for which there was information, the reduction in non-fatal myocardial infarction was highly significant (each $\mathrm{P}<0.001$ ), although the proportional reduction seemed to be greatest in patients treated early after acute myocardial infarction $\left(\chi^{2}\right.$ for heterogeneity between subcategories $=12.3, \mathrm{df}=3$; $\mathrm{P}=0.006$ ). But, even among patients who had not had an acute myocardial infarction, there was a clear reduc- 
Table 2 Effects of antiplatelet therapy on fatal and non-fatal strokes, subdivided by stroke aetiology

\begin{tabular}{|c|c|c|c|c|}
\hline \multirow[b]{2}{*}{ Category of trial } & \multicolumn{2}{|c|}{ No of fatal + No of non-fatal strokes/No of patients (combined \%) } & \multirow{2}{*}{$\begin{array}{l}\text { Stratified odds ratio } \\
\text { (SE) }\end{array}$} & \multirow{2}{*}{$\begin{array}{l}\text { Adjusted absolute } \\
\text { difference per } 1000 \text { (SE) }\end{array}$} \\
\hline & Antiplatelet groups & Adjusted controls & & \\
\hline \multicolumn{5}{|c|}{ Probable or definite haemorrhagic stroke (in trials with at least one haemorrhagic stroke) } \\
\hline Previous myocardial infarction & $6+5 / 5476(0.20)$ & $6+8 / 5507(0.25)$ & $0.8(0.4)$ & $-0.5(0.9)$ \\
\hline Acute myocardial infarction & $6+0 / 8821(0.07)$ & $1+1 / 8830(0.02)$ & $3.0(1.4)$ & $0.05(0.03)$ \\
\hline Previous stroke/transient ischaemic attack & $35+26 / 9553(0.64)$ & $26+28 / 9610(0.56)$ & $1.2(0.3)$ & $0.8(1.0)$ \\
\hline Acute stroke ${ }^{\star}$ & $68+140 / 20223(1.03)$ & $60+109 / 20205(0.84)$ & $1.2(0.1)$ & $1.9(0.9)$ \\
\hline Other high risk & $22+9 / 4498(0.69)$ & $9+14 / 4529(0.51)$ & $1.3(0.3)$ & $1.8(2.0)$ \\
\hline \multirow[t]{2}{*}{ Total } & $137+180 / 48571(0.65)$ & $102+160 / 48681(0.54)$ & $1.22(0.10)$ & \\
\hline & & & $\chi^{2}=2.5, \mathrm{df}=4 ; \mathrm{NS}$ & \\
\hline \multicolumn{5}{|c|}{ Probable or definite ischaemic stroke (in trials with at least one haemorrhagic stroke) } \\
\hline Previous myocardial infarction & $17+40 / 5476(1.04)$ & $17+65 / 5507(1.49)$ & $0.69(0.14)$ & $-4.5(2.1)$ \\
\hline Acute myocardial infarction & $8+29 / 8821(0.43)$ & $14+51 / 8830(0.75)$ & $0.58(0.15)$ & $-3.2(1.1)$ \\
\hline Previous stroke/transient ischaemic attack & $106+662 / 9553(8.04)$ & $136+876 / 9610(10.53)$ & $0.75(0.05)$ & $-24.0(5.1)$ \\
\hline Acute stroke & $90+231 / 20223(1.59)$ & $125+335 / 20205(2.28)$ & $0.69(0.06)$ & $-6.9(1.4)$ \\
\hline Other high risk & $45+110 / 4498(3.45)$ & $69+166 / 4529(5.19)$ & $0.63(0.09)$ & $-17.4(4.0)$ \\
\hline \multirow[t]{2}{*}{ Total } & $266+1072 / 48571(2.75)$ & $361+1493 / 48681(3.88)$ & $0.70(0.03)$ & \\
\hline & & & $\chi^{2}=3.3, \mathrm{df}=4 ; \mathrm{NS}$ & \\
\hline \multicolumn{5}{|c|}{ Strokes of any aetiology (in trials recording data on non-fatal strokes) } \\
\hline Previous myocardial infarction & $33+83 / 9222(1.26)$ & $51+129 / 9250(1.95)$ & $0.64(0.10)$ & $-6.9(1.9)$ \\
\hline Acute myocardial infarction & $13+32 / 9300(0.48)$ & $19+54 / 9291(0.58)$ & $0.62(0.15)$ & $-3.0(1.2)$ \\
\hline Previous stroke/transient ischaemic attack & $288+957 / 11493(10.83)$ & $314+1248 / 11527(13.55)$ & $0.77(0.04)$ & $-27.2(5.1)$ \\
\hline Acute stroke & $275+432 / 20238(3.49)$ & $293+522 / 20220(4.03)$ & $0.89(0.05)$ & $-5.4(1.9)$ \\
\hline Other high risk & $131+257 / 16607(2.34)$ & $152+364 / 16733(3.08)$ & $0.73(0.06)$ & $-7.5(1.7)$ \\
\hline \multirow[t]{2}{*}{ Total } & $740+1761 / 66860(3.74)$ & $829+2317 / 67021(4.75)$ & $0.78(0.03)$ & \\
\hline & & & $\chi^{2}=8.6, d f=4 ; P=0.07$ & \\
\hline
\end{tabular}

${ }^{*}$ Includes haemorrhagic transformation of original infarct.

tion of about one third in non-fatal myocardial infarction $(31 \%(4 \%) ; \mathrm{P}<0.0001)$.

\section{Stroke as outcome}

Information was available on 3522 non-fatal strokes after randomisation in 158 trials among high risk patients (compared with 1496 in 122 trials previously ${ }^{1}$ ) and on a further 1424 fatal strokes. Antiplatelet therapy produced a $25 \%(3 \%)$ proportional reduction in non-fatal stroke ( $\mathrm{P}<0.0001$, see bmj.com and fig 3), with no significant heterogeneity between the proportional reductions in the five high risk categories of patient $\left(\chi^{2}=5.8, \mathrm{df}=4\right.$; NS). Among those trials that recorded at least one haemorrhagic stroke, subdivision of all strokes (fatal or not) according to aetiology indicated that there was a proportional increase in fatal or non-fatal haemorrhagic stroke of 22\% (95\% confidence interval $3 \%$ to $44 \% ; \mathrm{P}<0.01)$ and a proportional decrease in fatal or non-fatal ischaemic stroke of $30 \%$ (24\% to $35 \%$; $\mathrm{P}<0.0001)$, with no significant heterogeneity between the proportional effects on each of these types of stroke in the five high risk categories studied $\left(\chi^{2}=2.5\right.$ and 3.3 respectively; both non-significant; table 2). But, although the proportional changes in the incidence of haemorrhagic and ischaemic stroke were about equal (and opposite) and although the absolute risks and benefits of antiplatelet therapy differed substantially from one category of patient to another, in each category the absolute risks were smaller than the benefits, so in each category of patient the overall risk of stroke (including strokes of unknown type) was reduced significantly (table 2 ).

Given these findings, the overall effect on total stroke may be estimated in any specific category of patient by considering the net absolute effects among such patients of the combination of an increase of about one quarter in the risk of haemorrhagic stroke and of a decrease of about one quarter in the risk of ischaemic stroke. So, for example, the proportional reduction in total stroke incidence was only around one half as large among patients with acute stroke as among patients in other high risk categories $\left(\chi^{2}=8.6\right.$, $\mathrm{df}=4 ; \mathrm{P}=0.07$; table 2 ) because in the month after an acute stroke about a quarter of the recurrent strokes in the control group were attributed to haemorrhage (or, particularly, to haemorrhagic transformation of the original infarct) whereas in other circumstances only about $6 \%$ were. Among all control patients (see totals in table 2), the case fatality rate is higher for haemorrhagic strokes (102 fatal, 160 not) than for ischaemic strokes (361 fatal, 1493 not). This may explain, at least in part, why the proportional effect of antiplatelet therapy on fatal strokes $(16 \% \quad(7 \%)$ reduction) seemed smaller, albeit non-significantly, than the effect on non-fatal strokes (28\% (4\%) reduction).

\section{Vascular and non-vascular deaths}

Information was available on 9605 deaths attributed to vascular (or unknown) causes in 193 trials among high risk patients (compared with 5253 in 141 trials previously ${ }^{1}$ ). Antiplatelet therapy produced a highly significant $15 \% \quad(2 \%)$ proportional reduction in vascular deaths $(\mathrm{P}<0.0001$; see bmj.com and fig 3$)$, with no significant heterogeneity between the proportional reductions in each of the five high risk categories of patient $\left(\chi^{2}=7.8, \mathrm{df}=4\right.$; NS).

A further 1414 deaths were attributed to nonvascular causes, but there was no excess of such death (785/71 $656(1.1 \%)$ antiplatelet $v 872 / 71876(1.2 \%)$ adjusted control; odds ratio $0.92,95 \%$ confidence interval 0.82 to 1.03 ; NS). Hence, antiplatelet therapy also produced a clear reduction of about one sixth in all cause mortality $(\mathrm{P}<0.0001$; see bmj.com $)$. If, 
Table 3 Effects of antiplatelet therapy on fatal and non-fatal major extracranial bleeds

\begin{tabular}{|c|c|c|c|c|}
\hline \multirow[b]{2}{*}{ Category of trial } & \multicolumn{2}{|c|}{$\begin{array}{l}\text { No of fatal }+ \text { No of non-fatal major bleeds/No of patients } \\
\text { (combined } \%)^{*}\end{array}$} & \multirow{2}{*}{$\begin{array}{l}\text { Stratified odds ratio } \\
\text { (SE) }\end{array}$} & \multirow{2}{*}{$\begin{array}{l}\text { Adjusted absolute } \\
\text { excess risk/1000 } \\
\text { (SE) }\end{array}$} \\
\hline & Antiplatelet groups & Adjusted controlst & & \\
\hline Previous myocardial infarction & $1+2 / 672(0.45)$ & $1+2 / 668(0.45)$ & - & - \\
\hline Acute myocardial infarction & $2+26 / 9134(0.31)$ & $3+20 / 9136(0.25)$ & $1.2(0.3)$ & $0(1)$ \\
\hline Previous stroke/transient ischaemic attack & $15+65 / 8276(0.97)$ & $7+32 / 8289(0.47)$ & $2.0(0.3)$ & $5(2) \ddagger$ \\
\hline Acute stroke & $60+135 / 20195(0.97)$ & $43+73 / 20178(0.57)$ & $1.7(0.1)$ & $4(1) \S$ \\
\hline Other high risk & $17+212 / 8881(2.58)$ & $17+135 / 8897(1.71)$ & $1.5(0.1)$ & $9(3) \ddagger$ \\
\hline Total & $95+440 / 47158(1.13)$ & $71+262 / 47168(0.71)$ & $1.6(0.1)$ ๆ & \\
\hline
\end{tabular}

*Only trials with systematic recording of all major extracranial bleeds (and that recorded at least one such bleed) are included.

†Percentage adjusted for unbalanced randomisation (see statistical methods).

$\ddagger \mathrm{P}<0.001$.

१ $\chi^{2}$ for heterogeneity $=2.6, \mathrm{df}=4$; NS

however, such antiplatelet therapy did have a protective or adverse effect on some specific cause of death (such as a particular type of cancer), the foregoing analysis of all non-vascular deaths might well be too insensitive to detect this. Site specific data on deaths from cancer were not available, so the suggestions that aspirin might prevent intestinal cancer ${ }^{14}$ or cause renal cancer ${ }^{15}$ could not be examined directly.

\section{Pulmonary embolism}

Only 32 trials planning to record symptomatic pulmonary embolism had recorded at least one non-fatal event, and among them antiplatelet therapy significantly reduced the risk of fatal or non-fatal pulmonary embolism (150/32 $777(0.46 \%)$ antiplatelet $v$ 200/ $32758(0.61 \%)$ adjusted control; odds reduction $25 \%$ $(10 \%) ; \mathrm{P}<0.01)$. In both the treatment group and the control group, about half of those who had a pulmonary embolism survived to the end of the trial. Hence, the risk reduction was about one quarter in both cases (although with wide confidence intervals). This proportional reduction is somewhat smaller than that found in the 1994 meta-analysis of trials among surgical and high risk medical patients $(47 / 4716$ $(1.0 \%)$ v 129/4730 (2.7\%); odds reduction $64 \%, 95 \%$ confidence interval $50 \%$ to $73 \% ; \mathrm{P}<0.0001)^{3}$ and in the subsequent pulmonary embolism prevention trial $(55 / 8726(0.6 \%)$ v $91 / 8718(1.0 \%)$; odds reduction $43 \%, 18 \%$ to $60 \% ; \mathrm{P}=0.002)$ among patients having hip or knee surgery. ${ }^{16}$

\section{Major extracranial bleeds}

Information was available on 787 major extracranial bleeds in 60 trials recording at least one such bleed. These were generally defined as bleeds that were fatal or required transfusion; among them, 159 (20\%) caused death. Little information was available on major extracranial bleeds from the trials of long term treatment after a myocardial infarction. Overall, the proportional increase in risk of a major extracranial bleed with antiplatelet therapy was about one half (odds ratio 1.6, 1.4 to 1.8), with no significant difference between the proportional increases observed in each of the five high risk categories of patient $\left(\chi^{2}=2.6\right.$, $\mathrm{df}=4$; NS; table 3$)$. The proportional increase in fatal bleeds was not significantly different from that for nonfatal bleeds, although only the excess of non-fatal bleeds was significant. There were too few fatal and non-fatal bleeds in any particular category to estimate the absolute risks directly. However, a useful estimate of the excess risk of a major extracranial bleed may be obtained indirectly by applying the proportional increase of about one half to the absolute risk of bleeding in that category of patients.

\section{Effects in different categories of high risk patients}

Patients with history of myocardial infarction

Among 18788 patients with a history of myocardial infarction in 12 trials (compared with 18573 such patients in 11 trials previously ${ }^{1}$ ), allocation to a mean duration of 27 months of antiplatelet therapy resulted in 36 (SE 5) fewer serious vascular events per 1000 patients (fig 2). This benefit reflects large and highly significant reductions in non-fatal reinfarction (18 (3) fewer per 1000; $\mathrm{P}<0.0001$ : fig $3 \mathrm{a}$ ) and vascular death (14 (4) fewer $/ 1000 ; \mathrm{P}=0.0006$ ) as well as a smaller, but still significant, reduction in non-fatal-stroke (5 (1) fewer $/ 1000 ; \mathrm{P}=0.002)$. These benefits were substantially larger than the excess risk of major extracranial bleeding, which was estimated indirectly from table 3 (as described above) to be about three additional major extracranial bleeds per 1000 patients allocated antiplatelet therapy-that is, an excess of about 1 such bleed per 1000 patients per year.

\section{Patients with acute myocardial infarction}

Data were available on 19288 patients with suspected acute myocardial infarction in 15 trials (compared with 18773 such patients in nine trials previously ${ }^{1}$ ), nearly all of whom were in the ISIS-2 trial. ${ }^{11}$ Allocation to a mean duration of one month of antiplatelet therapy resulted in 38 (5) fewer serious vascular events per 1000 treated patients (fig 2). This reflects large and highly significant reductions in non-fatal reinfarction (13 (2) fewer/1000; $\mathrm{P}<0.0001$ : fig $3 \mathrm{~b}$ ) and in vascular death (23 (4) fewer/1000; $\mathrm{P}<0.0001$ ), together with a small but significant reduction in non-fatal stroke (2 (1) fewer/1000; $\mathrm{P}=0.02)$. The net benefit is substantially larger than the excess risk of major extracranial bleeding (for example, from arterial lines), which was estimated to be about 1-2 additional major extracranial bleeds per 1000 patients allocated antiplatelet therapy.

\section{Patients with a history of stroke or transient ischaemic} attack

The amount of information available on the effects of prolonged antiplatelet therapy among patients with a history of stroke or transient ischaemic attack has increased substantially since 1990 (table 1). This is mainly because of the second European stroke preven- 
tion study, in which 6602 such patients were allocated to receive aspirin (25 $\mathrm{mg}$ twice daily), modified release dipyridamole (200 $\mathrm{mg}$ twice daily), both, or neither in a $2 \times 2$ factorial design. ${ }^{17}$ Overall, among 18270 patients in 21 trials (compared with 10255 patients in 18 trials previously $\left.^{1}\right)$, allocation to a mean duration of 29 months of antiplatelet therapy resulted in 36 (6) fewer serious vascular events per 1000 patients (fig 2). This benefit reflects a large and highly significant reduction in non-fatal stroke (25 (5) fewer/1000; $\mathrm{P}<0.0001$ : fig $3 \mathrm{c})$, along with a smaller but still significant reduction in non-fatal myocardial infarction (6 (2) fewer/1000; $\mathrm{P}=0.0009$ ).

Although the reduction in vascular mortality of 7 (4) per 1000 was only marginally significant $(P=0.04)$, the highly significant reductions in non-fatal vascular events and in all cause mortality (15 (5) fewer deaths/ $1000 ; \mathrm{P}=0.002)$ strongly reinforce the conclusion that prolonged antiplatelet therapy reduces the risk of death in such patients. These benefits clearly exceeded the estimated excess risk of bleeding of about 1-2 additional major extracranial bleeds per 1000 patients per year.

\section{Patients with acute ischaemic stroke}

Almost no information about the effects of antiplatelet therapy in acute ischaemic stroke was available for the previous analyses (table 1). ${ }^{1}$ Subsequently, results have emerged from the international stroke trial of $300 \mathrm{mg}$ daily aspirin versus open control ${ }^{18}$ and the Chinese acute stroke trial of $160 \mathrm{mg}$ daily aspirin versus placebo. $^{19}$ These trials each included about 20000 patients with suspected acute ischaemic stroke. ${ }^{20}$ Overall, among 40821 such patients in seven trials, allocation to a mean duration of three weeks of antiplatelet therapy produced an $11 \%(3 \%)$ proportional reduction in vascular events (fig 1), which was somewhat smaller than in other high risk categories. The resulting absolute reduction of 9 (3) fewer serious vascular event per 1000 patients (fig 2) reflects significant reductions in non-fatal stroke (4 (2) fewer/1000; $\mathrm{P}=0.003$; fig $3 \mathrm{~d}$ ) and in vascular deaths (5 (2) fewer/1000; $\mathrm{P}=0.05$ ), with information on non-fatal myocardial infarction not recorded in either the international stroke trial or Chinese acute stroke trial.

For 40428 patients in four of these trials, ${ }^{18} 192122$ it was possible to separate the stroke outcomes into those considered to be due to haemorrhage and those that were considered to be ischaemic (or of unknown cause). Antiplatelet therapy produced an absolute excess of 1.9 (SE 1.0) haemorrhagic strokes per 1000 patients, which was counterbalanced by an absolute reduction of 6.9 (1.4) fewer ischaemic strokes per 1000, yielding the overall reduction in the risk of any further stroke (including those of unknown cause) of 5.4 (1.9) per 1000. The excess risk of major extracranial

Fig 3 Absolute effects of antiplatelet therapy on various outcomes in patients with (a) previous myocardial infarction (12 trials); (b) acute myocardial infarction (15 trials); (c) previous stroke or transient ischaemic attack (21 trials); and (d) acute (presumed ischaemic) stroke (seven trials). Adjusted control totals have been calculated after converting any unevenly randomised trials to even ones by counting control groups more than once. In "any death" columns, non-vascular deaths are represented by lower horizontal lines (and may be calculated by subtracting vascular deaths from any deaths)

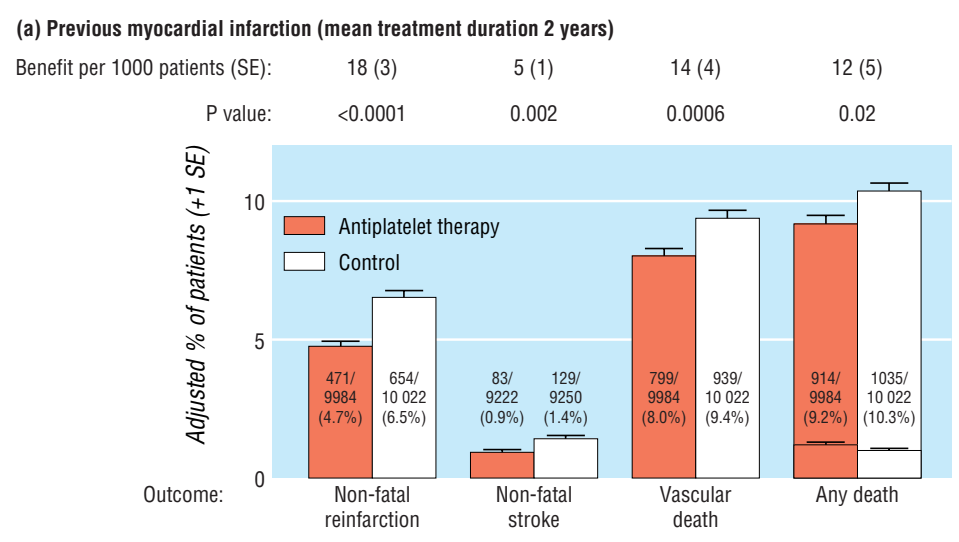

(b) Acute myocardial infarction (mean treatment duration 1 month)
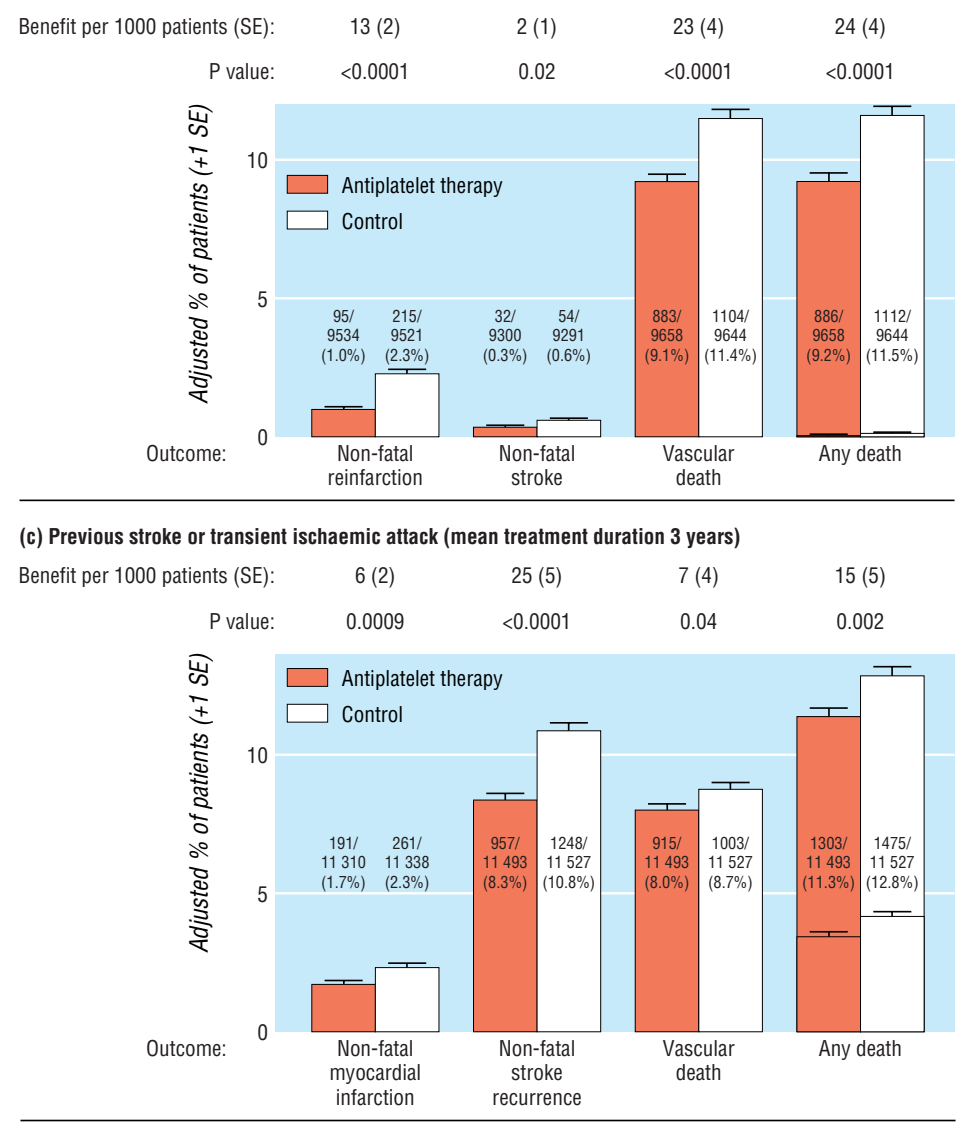

(d) Acute (presumed ischaemic) stroke (mean treatment duration 3 weeks)

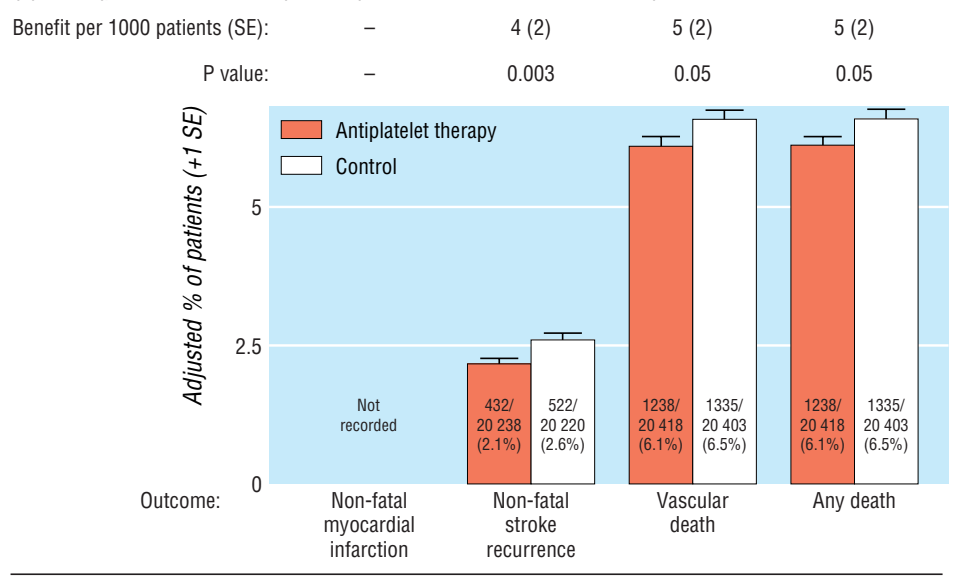




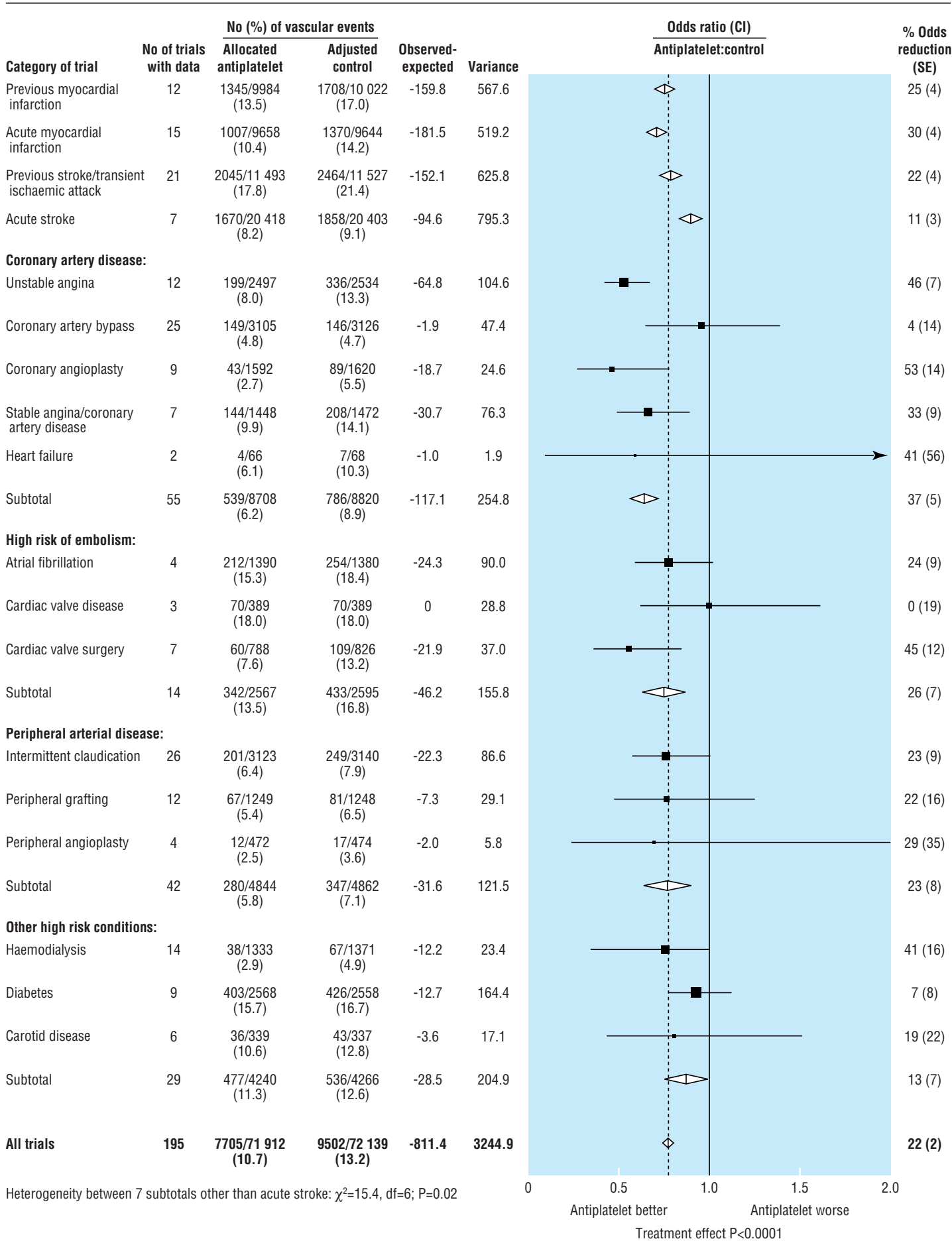

Fig 4 Proportional effects of antiplatelet therapy on vascular events in 195 trials in high risk patients subdivided by disease category. Stratified ratio of odds of an event in treatment groups to that in control groups is plotted for each group of trials (black square) along with its $99 \%$ confidence interval (horizontal line). Meta-analysis of results for each main category and for all trials (and $95 \%$ confidence interval) is represented by an open diamond. Adjusted control totals have been calculated after converting any unevenly randomised trials to even ones by counting control groups more than once, but other statistical calculations are based on actual numbers from individual trials

bleeding was estimated at about three additional such bleeds per 1000 allocated antiplatelet therapy, and more detailed analysis of the international stroke trial suggested that much of this excess occurred when antiplatelet therapy was given in conjunction with heparin (absolute excess 9 (2) per 1000 with heparin $v$ 2 (1) per 1000 without heparin). ${ }^{18}$
Effects in other high risk categories

In the previous cycle of analyses, ${ }^{1}$ information was available on serious vascular events from 104 trials among about 20000 patients with various other conditions associated with an increased risk of vascular events. Subsequently, more information has become available for certain of these conditions, which we have 
grouped into four main categories: coronary artery disease (which includes unstable angina, coronary artery bypass grafting, coronary angioplasty, stable angina, and heart failure); high risk of embolism (which includes non-rheumatic atrial fibrillation, cardiac valve disease, and cardiac valve surgery); peripheral arterial disease (which includes intermittent claudication, peripheral grafting, and peripheral angioplasty); and other high risk conditions (which includes haemodialysis patients having fistula or shunt placement, diabetes mellitus, and carotid disease).

In patients in each of the four main categories and their 14 components, the practical medical question is whether to give antiplatelet therapy. For that, it may suffice to know that the overall results for these "other high risk" categories show substantial reductions in myocardial infarction, stroke, and vascular death. This general conclusion can then be applied semiquantitatively in each of the 14 separate components, even if the results in one particular component considered separately do not indicate significant benefit.

Many of the odds ratios for individual components are associated with confidence intervals that are at least as wide as the likely magnitude of any treatment benefit (fig 4). Thus, even if antiplatelet therapy were of similar efficacy in all circumstances, several false negative results would be expected just from the play of chance. For example, the lack of evidence of benefit in patients randomised because of diabetes is not good evidence of lack of benefit in diabetic patients. (Indeed, even without any allowance for multiple comparisons, the $99 \%$ confidence interval for the proportional risk reduction in diabetic patients includes a risk reduction of one quarter, and the risks among diabetic patients are so high that the absolute benefit from such a risk reduction would be substantial.) Even if it is accepted, however, that antiplatelet treatment will produce an appreciable risk reduction in each type of patient at high risk of occlusive vascular disease, it is still of some interest to consider whether there is good evidence that the proportional risk reductions are different in different subcategories.

Other high risk patients with coronary artery disease Overall, among 15828 patients with coronary artery disease in 55 trials (compared with 9731 patients in 35 such trials previously ${ }^{1}$ ) there was a highly significant $37 \%(5 \%)$ proportional reduction in serious vascular events $(\mathrm{P}<0.0001$; fig 4$)$. The substantial increase in information available about the effects of antiplatelet therapy among patients with stable angina is due mainly to the results of the Swedish angina pectoris aspirin trial, in which 2035 patients were allocated to receive $75 \mathrm{mg}$ aspirin daily or placebo. ${ }^{23}$ There were independently significant benefits among patients with unstable angina $(46 \%(7 \%)$ reduction, $\mathrm{P}<0.0001)$, those having coronary angioplasty $(53 \% \quad(14 \%)$ reduction, $\mathrm{P}<0.0002)$, and those with stable angina $(33 \%(9 \%)$ reduction, $\mathrm{P}=0.0004)$.

The proportional risk reduction among patients who had recently had coronary artery bypass grafting was smaller $(4 \%(14 \%))$, but the confidence interval is wide and includes a risk reduction of one quarter. Hence, the apparent lack of effect of antiplatelet therapy on vascular events immediately after coronary artery bypass surgery may-given the clear evidence of benefit among other patients with coronary artery disease-be largely or wholly due to chance. Only 134 patients have been included in trials of antiplatelet therapy for heart failure, but most such patients have coronary artery disease, ${ }^{24}$ for which antiplatelet therapy is of known benefit.

\section{Patients at high risk of embolism}

Several cardiac and vascular conditions are associated with an increased risk of embolism to the brain or peripheral circulation, including atrial fibrillation (which was predominantly non-rheumatic), cardiac valve disease, and cardiac valve replacement. Overall, among 5162 patients at high risk of embolism in 14 trials (compared with 3190 such patients in nine trials previously $\left.^{1}\right)$ there was a highly significant $26 \%(7 \%)$ proportional reduction in serious vascular events $(\mathrm{P}=0.0003$; fig 4).

Atrial fibrillation is the commonest cardiac condition giving rise to embolism and is an important cause of stroke among elderly people. ${ }^{25}$ Most of the additional information about the effects of antiplatelet therapy among patients with atrial fibrillation was provided by the European atrial fibrillation trial, ${ }^{26}$ in which high risk patients with a previous stroke or transient ischaemic attack were randomised to aspirin or placebo (or oral anticoagulant, if eligible). Overall, among 2770 patients with atrial fibrillation in four trials there was a proportional reduction of $24 \%(9 \%)$ in serious vascular events (or 23\% (10\%) if one small trial of indobufen $v$ placebo that also included some patients without atrial fibrillation is excluded ${ }^{27}$ ).

\section{Patients with peripheral arterial disease}

Overall, among 9214 patients with peripheral arterial disease in 42 trials (compared with 4939 such patients in 33 trials previously ${ }^{1}$ ) there was a proportional reduction of $23 \%(8 \%)$ in serious vascular events $(\mathrm{P}=0.004$; fig 4$)$, with similar benefits among patients with intermittent claudication, those having peripheral grafting, and those having peripheral angioplasty (heterogeneity test $\chi^{2}=3.8, \mathrm{df}=3$; NS). Much of the new evidence came from the atherosclerotic disease evolution by picotamide trial, in which 2304 patients with intermittent claudication were allocated to receive the thromboxane synthase inhibitor picotamide or placebo. ${ }^{6}$

\section{Other high risk conditions}

Other groups of patients at high risk of occlusive arterial disease that have been studied include haemodialysis patients having shunt or fistula replacement, patients with diabetes, patients having carotid endarterectomy, and patients with asymptomatic carotid stenosis. Studies of antiplatelet therapy among haemodialysis patients after placement of a dialysis shunt or fistula included in the previous meta-analysis ${ }^{1}$ had typically lasted only a few weeks, but several of the more recent trials assessed the effects of 12-18 months of treatment. Overall, among 2632 patients in 14 trials (compared with only 525 patients in 10 trials previously ${ }^{1}$ ), antiplatelet therapy produced a $41 \%(16 \%)$ proportional reduction in serious vascular events. Even though this result is based on only 99 vascular events among such patients, it is consistent with the benefits seen in other circumstances. Chronic renal failure is associated with impaired haemostasis, but only 46 major extracranial bleeds (27/ 


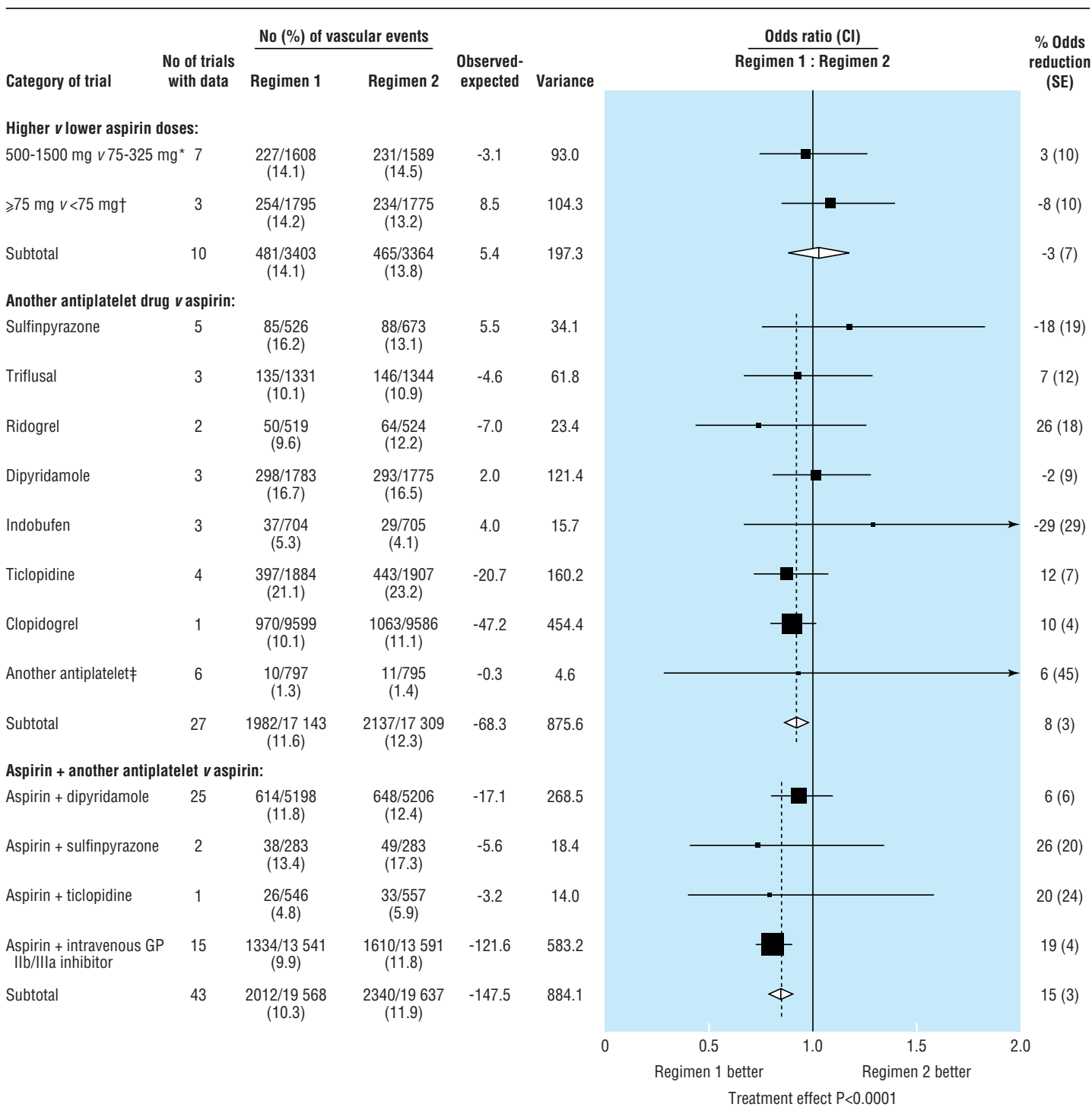

Fig 5 Direct comparisons of proportional effects of different antiplatelet regimens on vascular events in high risk patients. Only meta-analyses involving a total of 500 or more high risk patients are shown. *Includes one trial comparing $1400 \mathrm{mg} / \mathrm{day} v 350 \mathrm{mg} / \mathrm{day},{ }^{30}$ and another (excluding those with acute stroke) comparing $1000 \mathrm{mg} /$ day $\vee 300 \mathrm{mg} /$ day among patients who were also given dipyridamole. ${ }^{31}$ tIncludes two trials comparing $75-325 \mathrm{mg}$ aspirin daily $v<75 \mathrm{mg}$ aspirin daily ${ }^{32} 33$ and one trial of $500-1500 \mathrm{mg}$ aspirin daily $v<75 \mathrm{mg}$ aspirin daily.3. fIncludes cilostazol, sulotroban, trapidil, E5510, eptifibatide, and GR32191B. Stratified ratio of odds of an event in regimen 1 group to that in regimen 2 group is plotted for each group of trials (black square) along with its $99 \%$ confidence interval (horizontal line). Meta-analysis of results for all trials for a particular comparison (and 95\% confidence interval) is represented by an open diamond

$1333(2.0 \%)$ antiplatelet $v 31 / 1371(2.3 \%)$ adjusted control; NS) were recorded in these trials, so the size of any bleeding hazards cannot be reliably estimated.

Diabetes mellitus is also associated with an increased risk of vascular events, even in the absence of diagnosed cardiovascular disease. Much of the new information comes from the early treatment diabetic retinopathy study, ${ }^{28}$ in which 3711 people with diabetes (and, generally, no history of myocardial infarction or stroke) were allocated to receive $650 \mathrm{mg}$ aspirin daily or placebo. Overall, among 4961 patients with diabetes in nine trials (compared with 1365 patients in seven trials previously ${ }^{1}$, antiplatelet therapy was associated with only a $7 \%(8 \%)$ proportional reduction in serious vascular events (which remains consistent, however, with the reduction of about one quarter observed overall). None of the trials reported major extracranial bleeds, but the early treatment diabetic retinopathy study indicated that aspirin did not increase the risk of vitreous or retinal haemorrhage. ${ }^{29}$

Relatively small numbers of patients with carotid atherosclerosis have been studied. The overall results in five trials of antiplatelet therapy after carotid endarterectomy and one among patients with asymptomatic carotid disease (36/339 (10.6\%) antiplatelet $v$ 43/337 (12.8\%) adjusted control; $19 \%$ (22\%) reduction; NS) are consistent with those observed in other patients at high risk of stroke.

\section{Comparisons of different antiplatelet regimens}

Many small trials compared different antiplatelet drugs, but the analysis in figure 5 is restricted to direct randomised comparisons in which a total of at least 500 patients had been studied. The effects of different 


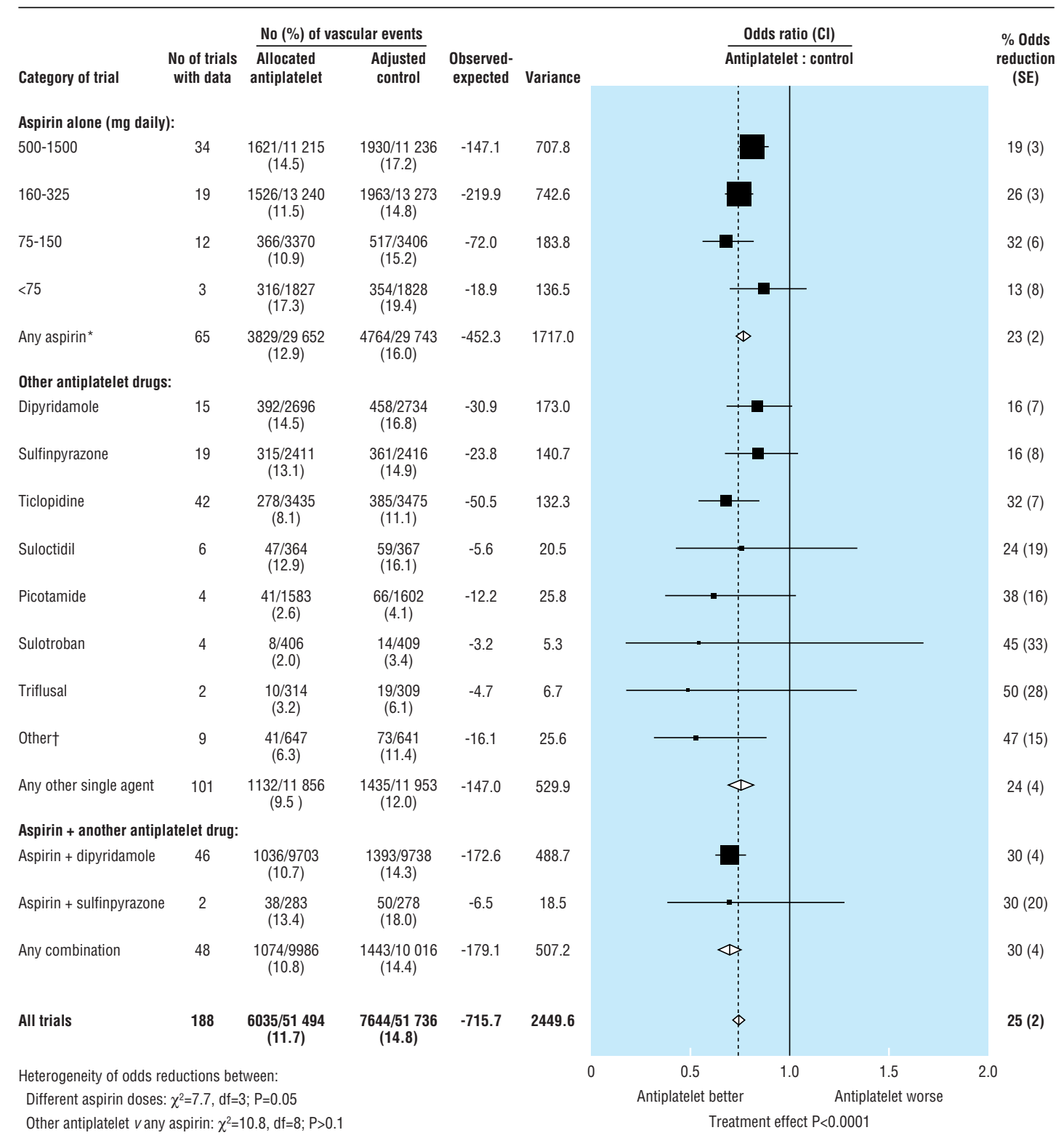

Fig 6 Indirect comparisons of proportional effects of different antiplatelet regimens on vascular events in high risk patients (excluding those with acute stroke). Only meta-analyses involving 500 or more high risk patients are shown. * Some trials contributed to more than one comparison. †Includes indobufen, flurbiprofen, GR32191B, dazoxiben, and trapidil. Stratified ratio of odds of an event in treatment groups to that in control groups is plotted for each group of trials (black square) along with its $99 \%$ confidence interval (horizontal line). Meta-analysis of results for each main comparison and for all trials (and 95\% confidence interval) is represented by an open diamond. Adjusted control totals have been calculated after converting any unevenly randomised trials to even ones by counting control groups more than once, but statistical calculations are based on actual numbers from individual trials

regimens can also be compared indirectly by comparing the size of the protective effect observed in the trials of one particular antiplatelet regimen versus control with the size of the protective effect in trials of another antiplatelet regimen versus control (fig 6). Such indirect comparisons need to be interpreted more cautiously than direct comparisons because there is some potential for bias if patients in the trials had different types of disease. Much of this bias can, however, be avoided by restricting attention to proportional reductions among high risk patients other than those with acute stroke (in whom antiplatelet therapy has a significantly smaller proportional effect on serious vascular events).
Effects of different doses of aspirin

Aspirin doses below $75 \mathrm{mg}$ daily have been suggested to be more effective than higher doses because such low doses are reported to "spare" prostacyclin (a platelet antiaggregant and vasodilator) and cause less gastrointestinal toxicity. ${ }^{5}$ Since the previous metaanalysis, much more information has become available from both direct and indirect comparisons on very low daily aspirin doses (table 1). Overall, among 3570 patients in three trials directly comparing aspirin $\geqslant 75$ $\mathrm{mg}$ daily $v$ aspirin $<75 \mathrm{mg}$ daily there was no significant difference between the different aspirin regimens (fig 5). However, aspirin doses of $<75 \mathrm{mg}$ have been less widely assessed than doses of 75-150 
mg daily, so there remains uncertainty about whether such low doses are as effective as daily doses of $\geqslant 75$ $\mathrm{mg}$. Among the trials of higher daily doses of aspirin $v$ no aspirin (fig 6), no particular range of aspirin dose was preferable for the prevention of serious vascular events. The proportional reduction in vascular events was $19 \%(3 \%)$ with $500-1500 \mathrm{mg}$ daily, $26 \%(3 \%)$ with $160-325 \mathrm{mg}$ daily, and $32 \%(6 \%)$ with $75-150 \mathrm{mg}$ daily. However, daily doses $<75 \mathrm{mg}$ seemed to have a somewhat smaller effect (proportional reduction 13\% (8\%); $\chi^{2}=7.7, \mathrm{df}=3 ; \mathrm{P}=0.05$; fig 6 ).

There was no good evidence to support the suggestion that aspirin doses of $\geqslant 1000 \mathrm{mg}$ daily might be preferable for the prevention of serious vascular events among patients at high risk of stroke. ${ }^{35}{ }^{36}$ This observation is reinforced by the aspirin and carotid endarterectomy trial (which was not included in this meta-analysis as it was reported after 1997). In that study the risk of the composite outcome of myocardial infarction, stroke, or death within three months of carotid endarterectomy was significantly lower among patients taking $81 \mathrm{mg}$ or $325 \mathrm{mg}$ aspirin daily than in those taking $625-1300 \mathrm{mg}^{37}$

In trials comparing aspirin with control, the proportional increase in the risk of a major extracranial bleed was similar with all daily aspirin doses $<325 \mathrm{mg}$ (odds ratios 1.7 (95\% confidence interval 0.8 to 3.3 ) for $<75 \mathrm{mg}$; 1.5 (1.0 to 2.3) for 75 -150 mg; and 1.4 (1.0 to 2.0) for $160-325 \mathrm{mg}$ ). Two trials that compared $75-325 \mathrm{mg}$ aspirin daily with $<75$ $\mathrm{mg}$ daily also found no significant difference in major extracranial bleeds $(39 / 1576(2.5 \%)$ with $75-325 \mathrm{mg} v$ 28/1555 (1.8\%) with < 75 mg; NS).

\section{Effects of antiplatelet drugs other than aspirin}

Overall, after trials among patients with acute stroke had been excluded, 81731 patients were included in 166 trials comparing a single antiplatelet drug with control among high risk patients (compared with 73218 patients in 141 trials previously ${ }^{1}$ ). Indirect comparisons of the different antiplatelet drugs provided no clear evidence of any differences in the effects on serious vascular events $\left(\chi^{2}\right.$ for heterogeneity between any aspirin regimen and the other antiplatelet drugs $=10.8, \mathrm{df}=8$; NS; fig 6), indicating that no large differences exist.

Direct randomised comparisons of different antiplatelet regimens could, if they were large enough, assess more reliably any modest differences that might exist. Most direct comparisons have assessed the effects of replacing aspirin with another antiplatelet (fig 5). Large scale randomised evidence was available only for clopidogrel versus aspirin. ${ }^{7}$ Overall, among 19185 patients with a history of myocardial infarction, stroke, or peripheral arterial disease, clopidogrel reduced serious vascular events by $10 \%(4 \%)$ compared with aspirin (970/9599 (10.1\%) clopidogrel v 1063/9586 $(11.1 \%)$ aspirin; $\mathrm{P}=0.03)$. This is similar to the $12 \%$ (7\%) reduction observed in trials of ticlopidine (a thienopyridine drug similar to clopidogrel) versus aspirin. The clopidogrel versus aspirin in patients at risk of ischaemic events trial also showed that the drug was relatively safe ${ }^{38}$ However, the true size of any difference between clopidogrel and aspirin could not be reliably estimated, since the $99 \%$ confidence interval ranged from a negligible benefit to a $20 \%$ further reduction in serious vascular events.
Effects of adding another antiplatelet drug to aspirin

Although the size of any difference between aspirin and other antiplatelet drugs may only be small, the addition to aspirin of an antiplatelet drug that acts through a different pathway might provide more substantial benefit than aspirin alone. The effects of adding dipyridamole, sulfinpyrazone, ticlopidine, or intravenous glycoprotein IIb/IIIa antagonists have been tested in randomised trials (fig 5).

Overall, among 10404 patients in 25 trials comparing dipyridamole plus aspirin with aspirin alone (compared with 5317 in 14 trials previously'), the addition of dipyridamole to aspirin was associated with only a non-significant further $6 \%(6 \%)$ reduction in serious vascular events $(614 / 5198(11.8 \%)$ aspirin plus dipyridamole $v 648 / 5206(12.4 \%)$ aspirin alone; fig 5). This overall result includes $183 \approx 236$ patients with non-fatal stroke, $150 v 134$ with non-fatal myocardial infarction, and 286 v 279 vascular deaths (with $5 v 1$ having both a non-fatal myocardial infarction and a non-fatal stroke). The apparent reduction in non-fatal stroke was derived mainly from one large study (109v 158), ${ }^{17}$ but this result was not supported by the findings for non-fatal stroke in the other studies (74v 78) or by the overall findings for non-fatal myocardial infarction or for vascular death.

Experimental and clinical studies have indicated that the platelet antiaggregatory effects of ticlopidine and aspirin may be additive. ${ }^{39}$ The combination has been studied among patients having coronary artery stenting, although mainly in non-randomised comparisons and case series. ${ }^{40}$ Several randomised trials have compared the combination of ticlopidine and aspirin with the combination of an oral anticoagulant and aspirin, but only two randomised trials have compared ticlopidine plus aspirin versus aspirin, and only one (the stent anticoagulation restenosis study ${ }^{41}$ ) was unconfounded. In that study, the addition of ticlopidine to aspirin was associated with a non-significant $21 \%(24 \%)$ further reduction in serious vascular events (fig 5). There was, however, a non-significant increase in major extracranial bleeds $(15 / 546(2.8 \%)$ ticlopidine plus aspirin $v 8 / 557(1.4 \%)$ aspirin alone). Further evidence that adding a thienopyridine to aspirin produces additional benefit among patients at acute risk of coronary occlusion has recently been provided by the clopidogrel in unstable angina to prevent recurrent events trial, ${ }^{42}$ but these results are not included as they were reported after September 1997.

The final common pathway of platelet aggregation is thought to be mediated by activation of platelet glycoprotein IIb/IIIa receptors by a platelet agonist (such as ADP, collagen, or thrombin) followed by crosslinking of activated receptors by circulating fibrinogen molecules. ${ }^{43}$ Drugs that block this receptor might therefore be especially effective. Many such drugs have now been developed, and in September 1997, 15 trials were available comparing aspirin plus a short (12-96 hour) intravenous infusion of a glycoprotein $\mathrm{Ilb} / \mathrm{III}$ antagonist with aspirin alone (one small study had studied an oral drug). Overall, among 24802 patients in these 15 trials, the addition of an intravenous glycoprotein $\mathrm{IIb} / \mathrm{III}$ a antagonist to aspirin produced a highly significant $19 \%(4 \%)$ proportional reduction in serious vascular events $(\mathrm{P}<0.0001)$, 
corresponding to the avoidance of about 20 vascular events per 1000 patients in just one month.

The proportional reduction in vascular events was significantly larger among patients having percutaneous coronary intervention than among patients not having such intervention $(32 \% \quad(6 \%)$ v $12 \% \quad(5 \%)$; heterogeneity test $\left.\chi^{2}=8.7, \mathrm{df}=1 ; \mathrm{P}=0.003\right)$. However, since patients with acute coronary syndromes not having a coronary procedure were at high risk of vascular events, addition of a glycoprotein IIb/IIIa antagonist was still associated with a worthwhile absolute benefit (15 vascular events avoided for 1000 treated during one month $\mathrm{P}<0.02)$. Overall, these benefits were offset by an absolute excess of 23 major extracranial bleeds per 1000 patients treated, although fatal bleeding was rare. The absolute risk of bleeding after percutaneous coronary intervention may be minimised by early removal of the sheath and reducing the dose of heparin. ${ }^{44}$ There were few intracranial haemorrhages (20/12 791 (0.2\%) glycoprotein IIb/IIIa antagonist plus aspirin $v 13 / 12833(0.1 \%)$ adjusted aspirin alone; NS).

\section{Discussion}

Since the previous meta-analysis, ${ }^{1}$ large amounts of information have become available from trials in patients having coronary artery procedures and in patients with acute stroke, stable angina, atrial fibrillation, peripheral arterial disease, and diabetes mellitus. Consequently, this analysis extends the direct evidence of benefit from antiplatelet therapy to a much wider range of patients at high risk of occlusive vascular disease. Antiplatelet therapy reduced the risk of serious vascular events (non-fatal myocardial infarction, non-fatal stroke, or vascular death) by about one quarter, not just among patients with unstable angina, acute myocardial infarction, stroke, or transient ischaemic attacks ${ }^{1}$ but also among other patients with coronary or peripheral arterial disease and those at high risk of embolism. Overall mortality was also significantly reduced in these high risk patients, and, compared with these benefits, the absolute risk of fatal and major nonfatal bleeds was small.

\section{Generalisability of findings to types of patients not studied directly}

Because these proportional risk reductions in vascular events were statistically reliable and seemed roughly homogeneous over the wide range of settings studied in these trials (fig 4 ) the protective effects of antiplatelet therapy should be expected to apply to an even wider range of high risk patients than those categories for which the present meta-analysis provides direct evidence of benefit. Thus, it would be inappropriate to base conclusions about the effects of antiplatelet therapy in each small subcategory of patients solely on the results from that subcategory. Compare, for example, the apparently contrasting effects on vascular events among patients having coronary angioplasty (in whom antiplatelet therapy seems to halve the risk) and those having coronary artery bypass grafting (in whom it seems to have no effect). Given the overall evidence for a reduction in serious vascular events of about one quarter among such a wide range of patients at high risk of occlusive vascular disease, it would not be reasonable to conclude that antiplatelet therapy halves vascular events after angioplasty but has no protective effect after coronary artery bypass surgery (particularly when antiplatelet therapy has been shown to produce a massively significant reduction in thrombotic occlusion of bypass grafts ${ }^{2}$ ).

Similarly, although antiplatelet therapy was associated with only a non-significant $7 \%(8 \%)$ proportional reduction in serious vascular events among patients with diabetes mellitus (but, predominantly, no history of myocardial infarction or stroke), these results do not provide reliable evidence of a lack of worthwhile benefit in such patients. Indeed, taken as a whole they indicate the converse, although direct evidence from further randomised trials of antiplatelet therapy among diabetic patients would still be helpful. However, our previous finding that antiplatelet therapy is similarly effective among patients with pre-existing symptomatic vascular disease who do and do not have diabetes ${ }^{1}$ suggests that aspirin is likely to be effective for the primary prevention of vascular events among diabetic patients. Furthermore, there is now good evidence that antiplatelet therapy is not associated with any special risks (such as bleeding in the eye) in patients with diabetes. ${ }^{29}$ Hence, it may be appropriate to consider antiplatelet therapy in diabetic patients who are at substantial risk of a first vascular event (such as those with proteinuria) ${ }^{45}$ and non-diabetic patients at high risk because of pre-existing vascular disease, even if there is no direct evidence of benefit (as for patients undergoing coronary artery surgery or those with heart failure ${ }^{46}$ ), provided that there are no special risks of bleeding that might outweigh the benefit. Thus, these findings can reasonably be extrapolated to a far wider range of high risk patients than those studied, but the further the extrapolation goes, the more desirable it is to have direct evidence-for example, for patients with renal disease, who are at high risk of myocardial infarction and ischaemic stroke ${ }^{47}$ but who also have special risks of bleeding.

\section{Acute stroke}

The randomised evidence that is available from about 40000 patients with acute stroke shows that, although antiplatelet therapy is associated with about two more major extracranial bleeds per 1000 treated in the absence of concomitant heparin, ${ }^{18}$ there will be about four fewer patients with a non-fatal stroke and five fewer patients dying from a vascular cause. Moreover, even among acute stroke patients who did not have computed tomography to exclude pre-existing cerebral haemorrhage before starting treatment, antiplatelet therapy seemed to produce net benefit. ${ }^{20}$ Hence, there is now good reason to consider starting antiplatelet therapy as soon as possible after suspected acute ischaemic stroke, preferably after confirmation by computed tomography (unless this would result in undue delay). This complements the previous evidence that continuing antiplatelet therapy for some years after the acute phase of ischaemic stroke produces substantial further reductions in risk.

\section{Benefits of different antiplatelet regimens}

The available data allow three main questions about treatment regimens to be examined: which range of aspirin doses seems most promising; is some other antiplatelet drug better than aspirin; and does any antiplatelet drug add to the net benefit of aspirin? 
Aspirin regimens

Within a few days of beginning $75 \mathrm{mg}$ aspirin daily, cyclo-oxygenase is virtually completely inhibited in platelets, producing an antithrombotic effect. ${ }^{5}$ The present analyses indicate that high doses of 500-1500 $\mathrm{mg}$ aspirin daily (which are more gastrotoxic ${ }^{48}$ ) are no more effective than medium doses of 160-325 mg/day or low doses of 75-150 mg/day (figs 5 and 6). Results from trials of lower doses are less conclusive. Hence, the available evidence supports daily doses of aspirin in the range $75-150 \mathrm{mg}$ for the long term prevention of serious vascular events in high risk patients. In clinical situations where an immediate antithrombotic effect is required (such as acute myocardial infarction, acute ischaemic stroke, unstable angina), a loading dose of about 150-300 mg, which is sufficient to produce rapid and complete inhibition of thromboxane mediated platelet aggregation, ${ }^{49}$ should probably be given.

\section{Other antiplatelet drugs}

Even though aspirin can prevent about one quarter of serious vascular events in a wide range of high risk patients, the residual risk may still be high. Hence, antiplatelet regimens are needed that are more effective than aspirin alone. Any real differences between two antiplatelet drugs are likely to be smaller than the differences between antiplatelet therapy and no antiplatelet therapy, so reliable comparisons between different drugs may require direct randomisation of many thousands, or even tens of thousands, of high risk patients. Such evidence exists only for clopidogrel versus aspirin, and this indicates that clopidogrel may be slightly more effective than aspirin (particularly when the evidence for ticlopidine, which is similar in structure and mechanism of action to clopidogrel, is also considered). ${ }^{50}$

Addition of other antiplatelet drugs to aspirin

Addition to aspirin of an antiplatelet drug that prevents platelet aggregation through some other pathway may well produce a further reduction in the risk of serious vascular events. This has now been shown for short term treatment. Large randomised trials among patients having percutaneous coronary interventions have found that adding a short intravenous infusion of glycoprotein IIb/IIIa antagonist reduces the risk of early arterial or stent thrombosis. ${ }^{43}$ In the present meta-analysis, evidence of benefit was limited to a follow up of only around one month, but recently published studies show that the benefit of glycoprotein $\mathrm{Ilb} / \mathrm{III}$ antagonists is maintained for at least six months (and possibly longer). ${ }^{51-53}$ Despite this, the oral IIb/IIIa-antagonists have not been found to add to the effects of aspirin..$^{54}$

Similarly, the addition of dipyridamole to aspirin has not been shown clearly to produce additional reductions in serious vascular events, although one trial suggested that there may be a worthwhile further reduction in stroke. ${ }^{17}$ Reasons for this apparent effect on stroke in that study include the possibility that the newer (and more bioavailable) formulation of dipyridamole was more effective than the older preparation. It is also plausible that these findings (which were not supported by other studies) arose largely or wholly by the play of chance, or were due to an insufficient daily aspirin dose or a slight antihypertensive effect of dipyridamole. Dipyridamole is being tested further in the European and Australian stroke prevention in reversible ischaemia trial..$^{55}$

Clopidogrel and ticlopidine, which are both thienopyridines, act by blocking ADP dependent activation of platelets. The effects of their antiplatelet properties on occlusive vascular events could therefore be complementary to those of aspirin, which inhibits thromboxane dependent activation. A large trial that assessed the effects of adding clopidogrel to aspirin among patients with unstable angina recently reported promising results, ${ }^{42}$ and the second Chinese cardiac study is assessing this question among patients with acute myocardial infarction. ${ }^{56}$ Long term studies of the effects of adding clopidogrel to aspirin might also be useful among other types of patients at high risk of occlusive vascular disease. Such studies could also examine the important question of whether adding clopidogrel is effective in patients who were taking aspirin when the event occurred (so called aspirin failures).

In the high risk setting of percutaneous coronary intervention or among high risk patients with an acute coronary syndrome, intensification of antiplatelet therapy by adding an intravenous glycoprotein IIb/IIIa antagonist or thienopyridine to aspirin may be appropriate. In other circumstances, however, aspirin at a dose of 75-150 mg daily is likely to be an appropriate antiplatelet regimen unless patients have a definite contraindication to aspirin-for example, definite allergy or appreciable gastric symptoms even with low dose aspirin. Clopidogrel might be an appropriate alternative in such patients.

\section{Benefits exceed hazards in most high risk patients}

Our results suggest that among individuals at high risk of occlusive vascular disease, the proportional risk reductions with antiplatelet therapy are roughly similar in most categories of patient (although they are smaller in acute stroke). Consequently, a patient's absolute risk is likely to be more important than the proportional reduction in serious vascular events in determining the likely benefit of antiplatelet therapy. In patients at particularly high risk of vascular events, the benefits of antiplatelet therapy are large. For example, among 1000 patients with acute myocardial infarction who are given one month of aspirin and then continue to take low dose aspirin for some years, about 40 would avoid a serious vascular event during the first month and about a further 40 would avoid a vascular event in the next couple of years. Similar sized long term benefits are likely to be seen if antiplatelet therapy is started soon after stroke or transient ischaemic attack and continued long term. Even in patient populations at intermediate risk (2-3\% a year of serious occlusive vascular events) such as some patients with no previous vascular event but with stable angina, atrial fibrillation, or peripheral arterial disease, antiplatelet therapy for a couple of years would be expected to prevent about 10-15 vascular events for every 1000 patients treated.

The present evidence suggests that the proportional increase in the risk of major bleeding of about one half is similar among a wide range of categories of patient. Population based observational studies have found that regular use of aspirin (at a dose of $\leqslant 300$ $\mathrm{mg}$ /day) is associated with around a twofold increased 
risk of upper gastrointestinal bleeding (or perforation). ${ }^{57}$ It therefore seems likely that the benefits of antiplatelet therapy will far outweigh any hazards unless the absolute risk of bleeding is high (such as among haemodialysis patients) or the absolute risk of a vascular event is low (as in apparently healthy people). Consequently, unless some definite contraindication exists, antiplatelet therapy should be considered routinely for all patients whose medical history implies a significant risk of occlusive vascular disease over the next few months or years, and it should generally be continued for as long as the risk remains high.

\section{Potential for wider use of antiplatelet therapy in high risk patients}

Recent audits have shown that the use of antiplatelet therapy has increased during the past few years but that a substantial proportion of high risk patients still do not receive it. For example, only about half (or less) of all patients with a history of myocardial infarction, angina, or peripheral arterial disease are currently receiving antiplatelet therapy, and rates tend to be lower in older people despite their higher absolute risk. ${ }^{58-60}$ Use of aspirin among patients with diabetes is even more limited, with one survey suggesting that less than a quarter of those with a clear history of coronary artery disease were taking regular aspirin, ${ }^{58}$ and another study finding that only $7 \%$ of those without a history of coronary artery disease were taking aspirin. ${ }^{60}$ Similarly, only about one third of patients with atrial fibrillation receive oral anticoagulants, the most effective treatment for the prevention of strokes in this condition. $^{61}{ }^{62}$ This may be because of the associated risks of bleeding and the need for anticoagulation monitoring. But less than half of such patients who are not taking anticoagulants receive antiplatelet therapy despite the high risk of stroke (especially in elderly people). ${ }^{63} 64$

These results reinforce the value of ensuring that antiplatelet therapy with 75-150 mg aspirin daily (or some other effective antiplatelet regimen) is considered routinely for all such patients at high or intermediate risk of occlusive vascular events (more than about $2 \%$ a year), irrespective of whether they have already had a major vascular event. An unanswered question, however, is whether it is possible to identify particular groups of apparently healthy people who may be at increased risk of myocardial infarction or stroke and for whom the benefits of daily aspirin outweigh the hazards. This is currently being investigated in an analysis of primary prevention trials. For most healthy individuals, however, for whom the risk of a vascular event is likely to be substantially less than $1 \%$ a year, daily aspirin may well be inappropriate.

This paper is dedicated to Gale Mead (1943-2001), who typed this and the previous reports from this collaboration.

Contributors: Writing committee: C Baigent, C Sudlow, R Collins, R Peto. Details of collaborators are available on bmj.com. The current cycle of this collaborative study was coordinated by $\mathrm{CB}$ and $\mathrm{CS}$. CB, CS, RC, and RP all contributed to drafting the manuscript, which was circulated to collaborators for comment and subsequent revision. The collaborators all provided trial data or other trial related information either in a previous cycle of the Antiplatelet Trialists' Collaboration or in the current cycle of the Antithrombotic Trialists' Collaboration. $\mathrm{CB}, \mathrm{CS}, \mathrm{RC}$, and $\mathrm{RP}$ are the study guarantors.

Funding: Since the inception of this collaboration several years ago, staff and computing have been provided by grants

\section{What is already known on this topic}

Antiplatelet therapy is effective for short term treatment of patients with suspected acute myocardial infarction and unstable angina

Long term treatment is beneficial for patients who have had a myocardial infarction, stroke, or transient ischaemic attack

Daily aspirin doses of 75-325 mg are effective

What this study adds

Antiplatelet therapy protects against vascular events among patients with stable angina, intermittent claudication, and (if oral anticoagulants are unsuitable) atrial fibrillation

Antiplatelet therapy can be started promptly during acute presumed ischaemic stroke and continued long term

Daily aspirin doses of 75-150 $\mathrm{mg}$ seem to be as effective as higher doses for long term treatments (and clopidrogel is an appropriate alternative for patients with a contraindication to aspirin)

Short term addition of a glycoprotein IIb/IIIa antagonist to aspirin prevents vascular events in patients having percutaneous coronary intervention and those with unstable angina but causes increased bleeding

from the Medical Research Council (United Kingdom), the Stroke Association, Chest, Heart and Stroke Scotland, Edinburgh University (Sir Stanley and Lady Davidson Fund); and by the Clinical Trial Service Unit and Epidemiological Studies Unit (Nuffield Department of Clinical Medicine, University of Oxford), which is supported by the Medical Research Council (United Kingdom), the British Heart Foundation, and the Imperial Cancer Research Fund. Support for the current cycle has also been contributed by a program grant from the European Union Biomed Programme (Contract number BMHCT93-1552 plus supplementary agreement number 2), and by a Wellcome Training Fellowship for C Sudlow.

Competing interests: The Clinical Trial Service Unit has received grants for independent research from AstraZeneca, Bristol-Myers Squibb, Hoffman-La Roche, Merck Sharp and Dohme, and Sanofi-Synthelabo. No grants were received from industry for the present cycle of analyses.

1 Antiplatelet Trialists' Collaboration. Collaborative overview of randomised trials of antiplatelet therapy. I. Prevention of death, myocardial infarction, and stroke by prolonged antiplatelet therapy in various categories of patients. BMJ 1994;308:81-106.

2 Antiplatelet Trialists' Collaboration. Collaborative overview of randomised trials of antiplatelet therapy. II. Maintenance of vascular graft or arterial patency by antiplatelet therapy. BMJ 1994;308:159-68.

3 Antiplatelet Trialists' Collaboration. Collaborative overview of randomised trials of antiplatelet therapy. III. Reduction in venous thrombosis and pulmonary embolism by antiplatelet prophylaxis among surgical and medical patients. BMJ 1994;308:235-46.

4 Sandercock PAG, van den Belt AGM, Lindley RI, Slattery J Antithrombotic therapy in acute ischaemic stroke: an overview of the completed randomised trials. J Neurol Neurosurg Psychiatry 1993;56:17-25.

5 Patrono C, Ciabattonni G, Patrignani P, Pugliese F, Filabozzi P, Catella F, et al. Clinical pharmacology of platelet cyclo-oxygenase inhibition. Circulation 1985;72:1177-84.

6 Balsano F, Violi F, ADEP Group. Effect of picotamide on the clinical progression of peripheral vascular disease. A double-blind placebocontrolled study. Circulation 1993;87:1563-9.

7 CAPRIE Steering Committee. A randomised, blinded, trial of clopidogrel versus aspirin in patients at risk of ischaemic events (CAPRIE). Lancet 1996;348:1329-39.

8 Rasmanis G, Vesterqvist O, Green K, Edhag O, Henriksson P. Effects of intermittent treatment with aspirin on thromboxane and prostacyclin 
formation in patients with acute myocardial infarction. Lancet 1988;ii:245-7.

9 Romeo F, Ruvolo G, Martuscelli E, Comito M, Cardona N, Colistra C, et al. Ticlopidine in the prevention of the block of aorto-coronary by-pass. In: Proceedings of satellite symposium of 83 rd congress of Italian Society of Internal Medicine. Rome: ISIM, 1982:155-60.

10 Early Breast Cancer Trialists' Collaborative Group. Treatment of early breast cancer. Vol 1. Worldwide evidence 1985-1990. Oxford: Oxford University Press, 1990:12-8.

11 ISIS-2 (Second International Study of Infarct Survival) Collaborative Group. Randomised trial of intravenous streptokinase, oral aspirin, both, or neither among 17187 cases of suspected acute myocardial infarction: ISIS-2. Lancet 1988;ii:349-60.

12 Yusuf S, Wittes J, Probstfield J, Tyroler HA. Analysis and interpretation of treatment effects in subgroups of patients in randomized clinical trials. JAMA 1991;266:93-8.

13 Collins R, MacMahon S. Reliable assessment of the effects of treatment on mortality and major morbidity. I. Clinical trials. Lancet 2001;357:373-80.

14 Thun MJ, Namboodiri MM, Heath Jr CW. Aspirin use and reduced risk of fatal colon cancer. $N$ Engl J Med 1991;325:1593-6.

15 Paganini-Hill A, Chao A, Ross RK, Henderson BE. Aspirin use and chronic diseases: a cohort study of the elderly. BMJ 1989:299:1247-50.

16 Pulmonary Embolism Prevention (PEP) Trial Collaborative Group. Prevention of pulmonary embolism and deep vein thrombosis with low dose aspirin: Pulmonary Embolism Prevention (PEP) trial. Lancet 2000;355:1295-302.

17 ESPS 2 Group. European stroke prevention study. 2. Efficacy and safety data. J Neurol Sci 1997;151(suppl):S1-77.

18 International Stroke Trial Collaborative Group. The International Stroke Trial (IST): a randomised trial of aspirin, subcutaneous heparin, both, or neither among 19435 patients with acute ischaemic stroke. Lancet 1997;349:1569-81

19 CAST (Chinese Acute Stroke Trial) Collaborative Group. CAST: randomised placebo-controlled trial of early aspirin use in 20000 patients with acute ischaemic stroke. Lancet 1997;349:1641-9.

20 Chen Z, Sandercock P, Pan H, Counsell C, Collins R, Liu L, et al. Indications for early aspirin use in acute ischemic stroke. A combined analysis of 40000 randomized patients from the Chinese acute stroke trial and the international stroke trial. Stroke 2000;31:1240-9.

21 Utsumi H. Evaluation of utility of ticlopidine, an antiplatelet agent, for acute cerebral infarction. Guildford: Sanofi Winthrop, 1984. (Sanofi internal report 001.6.128.)

22 Multicentre Acute Stroke Trial (MAST-I) Group. Randomised controlled trial of streptokinase, aspirin, and combination of both in treatment of acute ischaemic stroke. Lancet 1995;346:1509-14

23 Juul-Möller S, Edvardsson N, Jahnmatz B, Rosén A, Sørensen S, Ömblus $\mathrm{R}$, et al. Double-blind trial of aspirin in primary prevention of myocardial infarction in patients with stable chronic angina pectoris. Lancet 1992;340:1421-5

24 Massie BM, Shah NB. Evolving trends in the epidemiology of heart failure: a rationale for prevention. Am Heart J 1997;133:703-12.

25 Wolf PA, Abbott RD, Kannel WB. Atrial fibrillation as an independent risk factor for stroke: the Framingham study. Stroke 1991;22:983-8.

26 European Atrial Fibrillation Trial Study Group. Secondary prevention in non-rheumatic atrial fibrillation after transient ischaemic attack or minor stroke. Lancet 1993;342:1255-62.

27 Fornaro G, Rossi P, Mantica PG, Caccia ME, Aralda D, Lavezzari M, et al. Indobufen in the prevention of thromboembolic complications in patients with heart disease. A randomized, placebo-controlled, doubleblind study. Circulation 1993;87:162-4.

28 ETDRS Investigators. Aspirin effects on mortality and morbidity in patients with diabetes mellitus. Early treatment diabetic retinopathy study report 14.JAMA 1992;268:1292-300.

29 Chew EY, Klein ML, Murphy RP, Remaley NA, Ferris FL. Early Treatment Diabetic Retinopathy Study Research Group. Effects of aspirin on vitreous/preretinal hemorrhage in patients with diabetes mellitus. Arch Ophthalmol 1995;113:52-5.

30 Kadel C, Vallbracht C, Weidmann B, Kober G, Kaltenbach M. Aspirin and restenosis after successful PTCA: comparison of $1400 \mathrm{mg} v 350 \mathrm{mg}$ daily in a double-blind study [Abstract P2021]. Eur Heart J 1990;11(suppl):368.

31 Weichert W, Meents H, Abt K, Lieb H, Hach W, Krywanek JH, et al. Acetylsalicyclic acid-reocclusion-prophylaxis after angioplasty (ARPA-study). A randomized double-blind trial of two different dosages of ASA in patients with peripheral occlusive arterial disease. Vasa 1994;23:57-65.

32 Dutch TIA Trial Study Group. A comparison of two doses of aspirin (30 $\mathrm{mg} v 283 \mathrm{mg}$ a day) in patients after a transient ischemic attack or minor ischemic stroke. N Engl J Med 1991;325:1261-6.

33 Unité de Pharmacologie Clinique. ALDUSA (aspirin at low dose in unstable angina) pilot study. Report from the coordinating centre. Lyons: UPC, 1987.

34 Ranke C, Creutzig A, Luska G, Wagner HH, Galanski M, Bode-Boger S, et al. Controlled trial of high-versus low-dose aspirin treatment after percutaneous transluminal angioplasty in patients with peripheral vascular disease. Clin Invest Med 1994;72:673-80.

35 Dyken ML. Low-dose aspirin and stroke. "It ain't necessarily so." Stroke 1992;23:1395-9

36 Barnett HJM, Kaste M, Meldrum H, Eliasziw M. Aspirin dose in stroke prevention. Beautiful hypotheses slain by ugly facts. Stroke 1996;27:588-92.

37 Taylor DW, Barnett HJM, Ferouson GG, Sackett DL, Thorpe KE, Simard $\mathrm{D}$, et al. Low-dose and high-dose acetylsalicylic acid for patients undergoing carotid endarterectomy: a randomised controlled trial. Lancet 1999;353:2179-84.

38 Harker LA, Boissel JP, Pilgrim AJ, Gent M. Comparative safety and tolerability of clopidogrel versus aspirin. Results from CAPRIE. Drug Safety 1999;21:325-35.

39 Herbert JM, Lemat A, Samama M, Maffrand JP. The antiaggregating and antithrombotic activity of ticlopidine. Thromb Haemost 1996;76:94-8.
40 More RS, Chauhan A. Antiplatelet rather than anticoagulant therapy with coronary stenting. Lancet 1997;349:146-7.

41 Leon MB, Baim DS, Popma JJ, Gordon PC, Cutlip DE, Kalon KL, et al. A clinical trial comparing three antithrombotic-drug regimens after coronary-artery stenting. N Engl J Med 1998:339:1665-71.

42 CURE Study Investigators. Effects of clopidogrel in addition to aspirin in patients with non-ST segment elevation acute coronary syndromes. $N$ Engl J Med 2001;345:494-502.

43 Bhatt DL, Topol EJ. Current role of platelet glycoprotein IIb/IIIa inhibitors in acute coronary syndromes. JAMA 2000;284:1549-58.

44 Ferguson JJ, Kereiakes DJ, Adgey AAJ, Fox KAA, Willegass WB, Pfistere $\mathrm{M}$, et al. Safe use of platelet GP $\mathrm{Ilb} / \mathrm{III}$ a inhibitors. Eur Heart $J$ 1998:19:40-51

45 American Diabetes Association. Aspirin therapy in diabetes. Diabetes Care 1998;21:S45-6.

46 Massie BM. Antithrombotic therapy in heart failure: new perspectives. Eur Heart J 2000;2:E13-7.

47 Baigent C, Burbury K, Wheeler D. Premature cardiovascular disease in chronic renal failure. Lancet 2000;356:147-52.

48 Patrono C. Aspirin and human platelets: from clinical trials to acetylation of cyclooxygenase and back. Trends Pharmacol Sci 1989;10:453-8.

49 Reilly IAG, FitzGerald GA. Inhibition of thromboxane formation in vivo and ex vivo: implications for therapy with platelet inhibitory drugs. Blood 1987:69:180-6.

50 Hankey G, Sudlow C, Dunbabin DW. Thienopyridines versus aspirin to prevent stroke and other serious vascular events in patients at high risk of vascular disease? A systematic review of the evidence from randomized trials. Stroke 2000;31:1779-84.

51 Kong DF, Califf RM, Miller DP, Moliterno DJ, White HD, Harrington RA, et al. Clinical outcome of therapeutic agents that block the platelet glycoprotein $\mathrm{Ilb} / \mathrm{III} a$ integrin in ischemic heart disease. Circulation 1998;98:2829-35.

52 Lincoff AM, Califf RM, Topol EJ. Platelet glycoprotein IIb/IIIa receptor blockade in coronary artery disease. J Am Coll Cardiol 2000;35:1103-15.

53 Topol EJ, Ferguson JJ, Harlan F, Weisman MD, Tcheng JE, Ellis SG, et al. Long-term protection from myocardial ischemic events in a randomized trial of brief integrin $\beta 3$ blockade with percutaneous coronary intervention. JAMA 1997;278:479-84.

54 Heeschen C, Hamm CW. Difficulties with oral platelet glycoprotein $\mathrm{IIb} / \mathrm{III}$ receptor antagonists. Lancet 2000;355:330-1.

55 De Schryver ELLM, European/Australian Stroke Prevention in Reversible Ischaemia Trial (ESPRIT) Group. Design of ESPRIT: an international randomized trial for secondary prevention after non-disabling cerebral ischaemia of arterial origin. Cerebrovasc Dis 2000;10:147-50.

56 Second Chinese Cardiac Study (CCS-2) Collaborative Group. Rationale, design and organisation of the second Chinese cardiac study (CCS-2): a randomised trial of clopidogrel plus aspirin, and of metoprolol, amon patients with suspected acute myocardial infarction. J Cardiovasc Risk 2000;7:435-41.

57 García Rodríguez LA, Hernández-Diaz S, De Abajo FJ. Association between aspirin and upper gastrointestinal complications: systematic review of epidemiological studies. Br J Clin Pharmacol (in press).

58 Stafford RS. Aspirin use is low among United States outpatients with coronary artery disease. Circulation 2000;101:1097-101.

59 Campbell NC, Thain J, Deans HG, Ritchie LD, Rawles JM. Secondary prevention in coronary heart disease: baseline survey of provision in general practice. $B M J$ 1998;316:1430-4.

$60 \mathrm{MRC} / \mathrm{BHF}$ Heart Protection Study Collaborative Group. MRC/BHF heart protection study of cholesterol-lowering therapy and of antioxidant vitamin supplementation in a wide range of patients at increased risk of coronary heart disease: early safety and efficacy experience. Eur Heart J 1999;20:725-41.

61 Stafford RS, Singer D. Recent national patterns of warfarin use in atrial fibrillation. Circulation 1998;97:1231-3.

62 Sudlow M, Thomson R, Thwaites B, Rodgers H, Kenny RA. Prevalence of atrial fibrillation and eligibility for anticoagulants in the community. Lancet 1998;352:1167-71.

63 Lip GYH, Golding DJ, Nazir M, Beevers DG, Child DL, Fletcher RI. A survey of atrial fibrillation in general practice: the west Birmingham atrial fibrillation project. Br J Gen Practice 1997;47:285-9.

64 Bradley BC, Perdue KS, Tisdel KA, Gilligan DM. Frequency of anticoagulation for atrial fibrillation and reasons for its non-use at a Veterans Affairs Medical Center. Am J Cardiol 2000;85:568-72.

(Accepted 20 September 2001)

\section{Endpiece \\ Missed opportunity}

Everyone talks about changing the world. No one talks about changing himself.

Leo Tolstoy

Submitted by Peter Teichman, assistant professor, Department of Family Medicine, West Virginia University 